\title{
Non-Chromatographic Speciation of As by HG Technique-Analysis of Samples with Different Matrices
}

\author{
Maja Welna *, Anna Szymczycha-Madeja® and Pawel Pohl® \\ Department of Analytical Chemistry and Chemical Metallurgy, Faculty of Chemistry, \\ Wroclaw University of Science and Technology, Wybrzeze Wyspianskiego 27, 50-370 Wroclaw, Poland; \\ anna.szymczycha-madeja@pwr.edu.pl (A.S.-M.); pawel.pohl@pwr.edu.pl (P.P.) \\ * Correspondence: maja.welna@pwr.edu.pl; Tel.: +48-71-320-3232 \\ Academic Editor: Constantinos K. Zacharis \\ Received: 28 September 2020; Accepted: 24 October 2020; Published: 26 October 2020

\begin{abstract}
The applicability of the hydride generation (HG) sample introduction technique combined with different spectrochemical detection methods for non-chromatographic speciation of toxic As species, i.e., As(III), As(V), dimethylarsinate (DMA) and monomethylarsonate (MMA), in waters and other environmental, food and biological matrices is presented as a promising tool to speciate As by obviating chromatographic separation. Different non-chromatographic procedures along with speciation protocols reported in the literature over the past 20 year are summarized. Basic rules ensuring species selective generation of the corresponding hydrides are presented in detail. Common strategies and alternative approaches are highlighted. Aspects of proper sample preparation before analysis and the selection of adequate strategies for speciation purposes are emphasized.
\end{abstract}

Keywords: arsenic; arsenic species; hydride generation; atomic spectrometry; non-chromatographic speciation; selective reduction; speciation protocols; environmental; food and biological matrices; sample preparation

\section{Introduction}

Because the toxicity and physiological behavior of various As compounds differ greatly, knowledge regarding As species is crucial to understand their potential harmful effects to human beings. Arsenic has a variety of inorganic and organic forms ranging from highly hazardous inorganic arsenicals (i-As), i.e., arsenite (As(III)) and arsenate $(\mathrm{As}(\mathrm{V}))$, to relatively less toxic methyl-substituted organic arsenicals (o-As), i.e., monomethylarsonate (MMA) and dimethylarsinate (DMA). Other o-As compounds, e.g., arsenobetaine (AsB), arsenocholine (AsC) and As-sugars, typically present in marine organisms, are generally considered to be non-toxic [1]. Accordingly, today, determination of the total As content is insufficient, and speciation information is essential to reflect the risk associated with exposure to this element.

As shown in Table 1, contamination with As can be found in various environment compartments including atmosphere (as gaseous compounds and in particulate matter), water, rocks, soil and plants [2-59]. It can be emitted naturally from volcanic activity or from anthropogenic sources, such as mines, coal-fired plants for energy production, pesticides, phosphate fertilizer factories, irrigation and oxidation of volatile arsines in air, dust from burned fossil fuels as well as the disposal of industrial, municipal and animal waste $[32,34]$. The widespread use of phosphate rocks in the production of phosphate fertilizers is a significant source of As contamination and cause of exposure to this element [33,36]. The tendency of As to accumulate in plant materials, e.g., cereals, tea plants, vegetables, fruits or herbs, causes it to subsequently appear in food products, beverages or (natural) pharmaceuticals 
(see Table 1). Among them, rice (together with water) is considered to be the highest contributor to i-As intake among all products of vegetable origin. Although AsB and As-sugars are major components $(>90 \%)$ in seafood, e.g., fish (AsB) [52,53] and seaweed, e.g., algae (As-sugars) [44,53-55], the level of minor i-As, which is responsible for As toxicity in these samples, should also be monitored. Therefore, analyses of the contents of toxic As species in various environmental and food samples would provide information about their quality and safety. On the other hand, analyses of biological/clinical samples like human urine, tissue, cells or blood [4,31,60-63] would be helpful in recognizing As species in body fluids and tissue to evaluate the pattern of i-As metabolism and assess the risk of adverse health effects associated with i-As exposure. It is worth mentioning that As was also applied historically as a medicinal treatment [56]; nowadays, it is used, e.g., in glass production as a fining agent [35]. 
Table 1. The concentrations of As species in different samples obtained by non-chromatographic methods based on the hydride generation technique a

\begin{tabular}{|c|c|c|c|c|c|c|}
\hline Matrix & As(III), $\mu \mathrm{g} \mathrm{L}^{-1}$ & $\operatorname{As}(\mathrm{V}), \mu \mathrm{g} \mathrm{L}^{-1}$ & $\mathrm{i}-\mathrm{As}, \mu \mathrm{g} \mathrm{L}^{-1}$ & DMA, $\mu \mathrm{g} \mathrm{L}^{-1}$ & MMA, $\mu \mathrm{g} \mathrm{L}^{-1}$ & $o-A s{ }^{b}, \mu g^{-1}$ \\
\hline \multicolumn{7}{|c|}{ Liquid Matrices } \\
\hline \multicolumn{7}{|c|}{ Waters } \\
\hline drinking & $0.17-4.7[2-4]$ & $0.05-63.9[2-5]$ & & & & \\
\hline ground & $0.080-395[4,6-11]$ & $0.01-312[4,6-11]$ & $0.31-308[12-14]$ & & & \\
\hline underground & $1.25-1016[3,15,16]$ & $0.97-554.3[3,15,16]$ & & $1.4-2.8[3]$ & $0.8-1.2[3]$ & \\
\hline tap & $0.015-12.7[4,9,16-19]$ & $0.050-35[4,9,15-21]$ & & & & $0.30-0.80$ [19] \\
\hline river & $0.235-1.4[4,10,20,22]$ & $0.186-2.22[4,10,20-22]$ & $1.79-2.05[14]$ & & & \\
\hline lake & $0.11-0.95[10,20,23,24]$ & $0.03-1.30[10,20-24]$ & $3.80[14]$ & $0.007[24]$ & & \\
\hline sea & $0.03-2.2[3,10,11,17,24,25]$ & $0.17-19.8[3,10,11,17,24,25]$ & & $0.15[24]$ & $0.15-0.19[3]$ & \\
\hline waste & $0.345[4]$ & $0.052-0.957[4,21]$ & & & & \\
\hline rain & & $1.52[26]$ & $5.16[26]$ & & & \\
\hline snow & & $2.04[26]$ & $3.60[26]$ & & & \\
\hline \multicolumn{7}{|c|}{ Beverages } \\
\hline wine & $1.3-21.3[27,28]$ & & & & & \\
\hline tea & $0.3-14.4[29]$ & $56-59.6[29]$ & & & & $11.4-23.3$ [29] \\
\hline fruit juices & $0.3-3.9[30]$ & $0.12-6.6[30]$ & & & & \\
\hline \multicolumn{7}{|c|}{ Biological Samples (human fluids/tissue) } \\
\hline serum & $0.604-0.838[4]$ & $1.087-3.010[4]$ & & & & \\
\hline urine & $0.548-3.142[4]$ & $0.410-1.334[4]$ & & & & \\
\hline blood/blood plasma & & & $15.8-19.2[31]$ & $13.4-34.8[31]$ & $13.5-30.6[31]$ & \\
\hline \multicolumn{7}{|c|}{ Solid Matrices } \\
\hline Matrix & As(III), ng g $^{-1}$ & As(V), $\mathrm{ng} \mathrm{g}^{-1}$ & i-As, $\operatorname{ng~g}^{-1}$ & $\mathrm{DMA} \mathrm{ng} \mathrm{g}^{-1}$ & MMA, ng g ${ }^{-1}$ & $\mathrm{o}-\mathrm{As}^{\mathrm{b}}, \mathrm{ng} \mathrm{g}^{-1}$ \\
\hline \multicolumn{7}{|c|}{ Environmental } \\
\hline soil & $5.2-8.1^{\mathrm{c}}[32]$ & $16.0-20.4^{\mathrm{c}}[32]$ & & & & \\
\hline phosphate rocks & $2.1-3.9 \mu \mathrm{g} \mathrm{g}^{-1}[33]$ & & $5.2-20.0 \mu \mathrm{g} \mathrm{g}^{-1}[33]$ & & & \\
\hline airborne particulate matter & $2.7-10.5 \mathrm{ng} \mathrm{m}^{-3}[34]$ & & $3.8-20 \mathrm{ng} \mathrm{m}^{-3}[34]$ & & & \\
\hline \multicolumn{7}{|c|}{ Industrial } \\
\hline glass & $13.6-395 \mu \mathrm{g} \mathrm{g}^{-1}[35]$ & $10.6-1205 \mu \mathrm{g} \mathrm{g}^{-1}[35]$ & & & & \\
\hline
\end{tabular}


Table 1. Cont.

\begin{tabular}{|c|c|c|c|c|c|c|}
\hline Matrix & $\operatorname{As}(\mathrm{III}), \mu \mathrm{g} \mathrm{L}^{-1}$ & $\operatorname{As}(V), \mu \mathrm{g} \mathrm{L}^{-1}$ & i-As, $\mu \mathrm{g} \mathrm{L}^{-1}$ & DMA, $\mu \mathrm{g} \mathrm{L}^{-1}$ & MMA, $\mu \mathrm{g} \mathrm{L}^{-1}$ & $o-A s^{b}, \mu g L^{-1}$ \\
\hline \multicolumn{7}{|c|}{ Agricultural Agents } \\
\hline phosphate fertilizers & $2.6-7.5 \mu \mathrm{g} \mathrm{g}^{-1}[33,36]$ & & $\begin{array}{c}11.79-69.02 \mu \mathrm{g} \mathrm{g}^{-1} \\
{[33,36]}\end{array}$ & & & \\
\hline $\begin{array}{l}\text { pesticide } \\
\text { herbicyde }\end{array}$ & $0.90 \mu \mathrm{g} \mathrm{g}^{-1}[37]$ & $\begin{array}{l}0.81 \mu \mathrm{g} \mathrm{g}^{-1}[37] \\
1.47 \mathrm{~g} \mathrm{~g}^{-1}[37]\end{array}$ & & & & \\
\hline \multicolumn{7}{|c|}{ Food } \\
\hline rice & $22-248[22,38-40]$ & $5-76[22,38-40]$ & $30-600[41-46]$ & $\begin{array}{c}4.2-67.3 \\
{[22,39,40]}\end{array}$ & $2.2-38.1[22,39]$ & $12.2-112.2[45]$ \\
\hline rice products & $12.3-52.7[47]$ & $1.4-29.6[47]$ & $20-570[41-48]$ & $15-89[48]$ & $2.9-5.3[48]$ & \\
\hline wheat semolina & 55 [39] & 25 [39] & & $1.9[39]$ & $1.5[39]$ & \\
\hline milk & $1.5-5.9[49]$ & $2.1-8.1[49]$ & & & & \\
\hline mushrooms & $81-624[50]$ & $59-380[50]$ & & & & \\
\hline chard & $89.2-90.6[51]$ & $14.2-15.3[51]$ & & $4.1-4.3[51]$ & $3.5-3.7[51]$ & \\
\hline aubergine & $20.6-20.9[51]$ & $61.0-61.9[51]$ & & $1.1-1.2[51]$ & $1.2[51]$ & \\
\hline \multicolumn{7}{|c|}{ Marine Organism } \\
\hline fish & $80.3-230[52]$ & $108-310[52]$ & & 510-1310 [52] & $490-780[52]$ & $9.4-16^{\mathrm{a}, \mathrm{c}} \mu \mathrm{g} \mathrm{g}^{-1}[52]$ \\
\hline seaweed & & & $\begin{array}{c}0.05-57.5, \mu \mathrm{g} / \mathrm{g} \\
{[44,53-55]}\end{array}$ & & & \\
\hline \multicolumn{7}{|c|}{ Pharmaceuticals } \\
\hline TCM (herbs) & $22.8^{\mathrm{c}}[56]$ & $145.1^{\mathrm{c}}[56]$ & & & & $116.4^{\mathrm{b}, \mathrm{d}}[56]$ \\
\hline $\begin{array}{l}\text { herbaceous plant } \\
\text { dietary supplements }\end{array}$ & $\begin{array}{c}0.030-8.32 \mu \mathrm{g} \mathrm{g}^{-1}[57,58] \\
25-93[59]\end{array}$ & $\begin{array}{c}0.050-4.59 \mu \mathrm{g} \mathrm{g}^{-1}[57] \\
58-201[59]\end{array}$ & $1.08-6.91 \mu \mathrm{g} \mathrm{g}^{-1}[58]$ & & & $0.040, \mu \mathrm{g} \mathrm{g}^{-1}$ \\
\hline
\end{tabular}


Speciation analysis involves two steps, i.e., separation of different forms and their subsequent quantification. The most common and recommended approach providing complete information on the distribution of species and their structures employs chromatographic separation of As species by high performance liquid chromatography (HPLC) interfaced with inductively coupled plasma mass spectrometry (ICP-MS) due to extremely low detection limits (LODs) of As species and their high selectivity [64].

Concurrently to HPLC-based As speciation schemes, there is considerable interest in developing simpler, more robust and reliable non-chromatographic methodologies for the determination of toxic As forms in different matrices at trace levels. Among them, hydride generation (HG) is a promising alternative to speciate As by obviating the need for chromatography separation. The generation of volatile hydrides in reactions with sodium/potassium tetrahydroborate $\left(\mathrm{NaBH}_{4} / \mathrm{KBH}_{4}\right)$ in acidic media (usually $\mathrm{HCl}$ ) is a well-known derivatization sample introduction technique applied in parallel with atomic and/or mass detection methods for the determination of trace levels of As. It can be easily coupled with various detectors like atomic absorption spectrometry (AAS), atomic fluorescence spectrometry (AFS), inductively coupled plasma optical emission spectrometry (ICP-OES) and ICP-MS, providing selectivity, sensitivity, detectability, separation of analytes from a sample matrix and efficiency that exceeds that offered by conventional pneumatic sample nebulization (PN) [65].

The HG technique, originally developed to separate As from the sample matrix, was found to be a viable method by which to discriminate among major toxic As compounds, i.e., i-As, DMA and MMA. Satisfactorily, all these forms are hydride-active and react with $\mathrm{NaBH}_{4}$, forming the corresponding hydrides (arsines), i.e., $\mathrm{AsH}_{3},\left(\mathrm{CH}_{3}\right)_{2} \mathrm{AsH}$ and $\mathrm{CH}_{3} \mathrm{AsH}_{2}$ for $\mathrm{As}(\mathrm{III}, \mathrm{V})$, DMA and MMA [66,67]. Other arseno-organic compounds like AsB and AsC or As-sugars are not reducible and are decomposed to i-As, usually by microwave [51,52] or UV-irradiation, prior to HG [19,68]. However, recent developments have shown that significant HG activity of As-sugars can be achieved under properly chosen conditions in a batch reaction mode [69].

The effectiveness of the HG processes of these individual As forms differs largely and depends strongly on experimental conditions used, i.e., basically, the type and concentration of acid, as well as the $\mathrm{pH}$ of the reacting medium. Generally, $\mathrm{As}(\mathrm{III})$ can be reduced in a wide $\mathrm{HCl}$ concentration range ( $\mathrm{pH} 0-9)$, while for $\mathrm{As}(\mathrm{V})$, strong acidity is required $(\mathrm{pH}<2)[5,6,70]$. In contrast, at low concentrations of $\mathrm{HCl}$, both DMA and MMA hydrides can be effectively generated [71]. Trivalent arsenic can be also converted to arsine in the presence of weak organic acids (citric, acetic, tartaric) [3,40,71,72]. Moreover, this condition could also be applied to HG for DMA and MMA, but not for As(V) [3,71]. Nevertheless, there are considerable differences between the sensitivities obtained for the $\mathrm{i}$-As and o-As forms, and the HG efficiency for V-valent As species is lower than that for As(III) [3,27,38,53,71]. Therefore, finding a compromise reaction medium under which the same response can be obtained for all four As species is problematic. Thus, a pre-reduction step is usually carried out with suitable pre-reductants (mostly KI-ascorbic acid, thiourea and L-cysteine) to ensure that any inorganic and methyl-substituted As(V) forms are present as $\mathrm{As}(\mathrm{III})$ before their reaction with $\mathrm{NaBH}_{4}[3,40,71]$. However, similar responses of $\mathrm{As}(\mathrm{III})$ and $\mathrm{As}(\mathrm{V})$ species under appropriately selected reaction conditions can be achieved, and hence, pre-treatment before HG can be avoided [20,35,72].

On the other hand, the ability of the HG technique to differentiate As forms by their oxidation state (III/V) or nature (inorganic/organic) using simple procedures certainly broadens the application range of HG regarding As speciation, without the use of chromatographic separation (see Table 1). Non-chromatographic approaches to differentiating the four As species are less time-consuming, simpler and more suitable and affordable compared to HPLC $[64,73,74]$. Generally, selective hydride formation can be achieved by adjusting the reducing conditions in terms of the reaction medium, the $\mathrm{NaBH}_{4} / \mathrm{HCl}$ concentration and the sample pre-treatment with suitable additives. As a result, based on different responses of As species resulting from HG, non-chromatographic protocols for As speciation are proposed with variants for determinations of two- (e.g., [2,12,50,72]), three- (e.g., [7,75,76]) and four- (e.g., $[3,28,39,40])$ species of As in one sample. 
The speciation of As by HG in sample solutions containing various inorganic and organic species is challenging, because several factors affecting the efficiency have to be carefully controlled to improve the accuracy. In reference to this, the present review covers the speciation of As in various matrices including environmental, industrial, food, biological and clinical samples using the non-chromatographic approach, based on the HG technique, and combined with different spectrochemical detection methods. It illustrates the main aspects of proper sample preparation before analysis and the choice of an adequate strategy for speciation (see Figure 1). Analytical methodologies for As speciation by HG are categorized according to their analytical performance, advantages and problems. Different non-chromatographic procedures, along with the speciation protocols reported in the literature over the past 20 year, are summarized. Additionally, the role of specific extraction-complexation, retention and co-precipitation enrichment methods before HG, in addition to the separation of evolved hydrides in cold traps or pervaporation modules to improve the detectability of As species and the selectivity of measurements, are discussed. Finally, alternative methods to conventional wet chemical HG providing selective generation of As hydrides are presented.

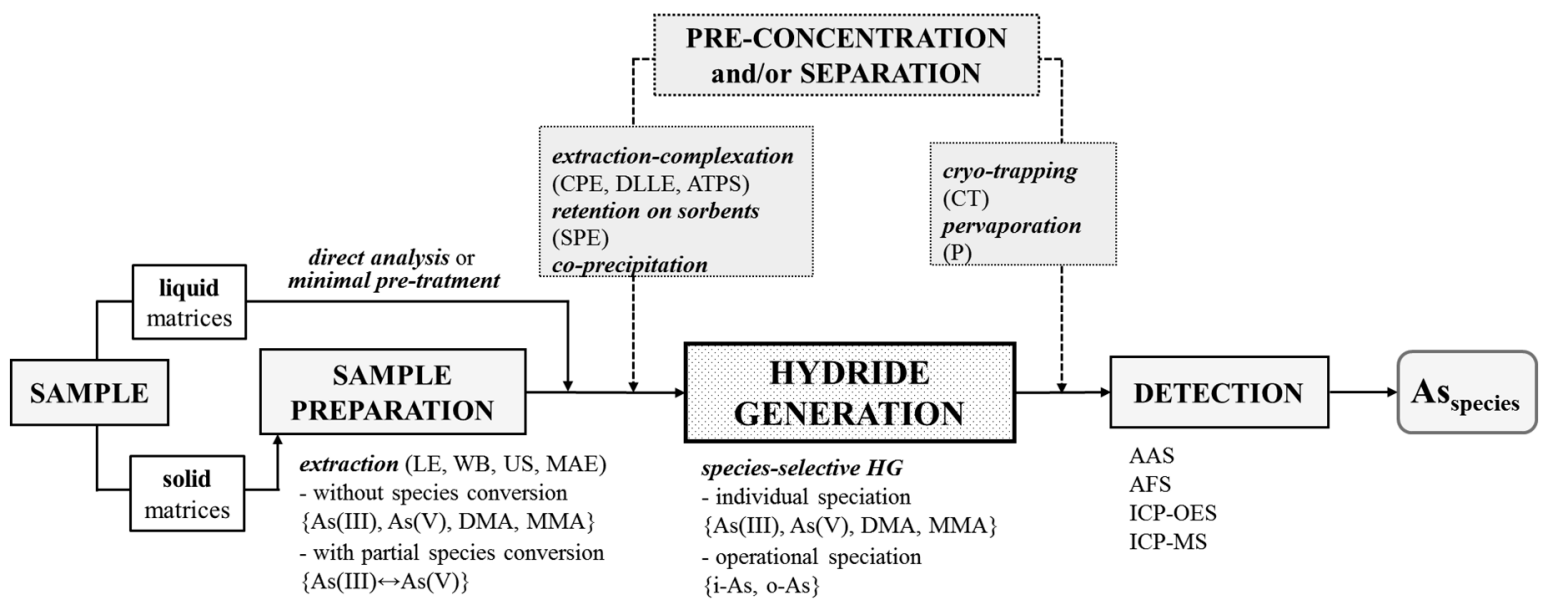

Figure 1. Analytical methodology for non-chromatographic speciation of As by HG technique. AAS: atomic absorption spectrometry. AFS: atomic fluorescence spectrometry. ATPS: liquid-liquid extraction of aqueous two-phase systems. CPE: cloud point extraction. CT: cryogenic cold trapping separation technique. DLLME: dispersive liquid-liquid microextraction. ICP-OES: inductively-coupled plasma optical emission spectrometry. ICP-MS: inductively-coupled plasma mass spectrometry. i-As: the inorganic tri- and penta- valent As species (As(III) and As(V)). LE: solvent extraction. MAE: microwave-assisted extraction. o-As: the organic, i.e., methylated pentavalent As species (DMA and MMA). P: pervaporation-based membrane separation technique. SPE: solid phase extraction. US: ultrasonication in an ultrasound water bath. WB: water bath.

\section{Instrumental Techniques Used for the Determination of As Species and Ways of Verifying the Reliability of the Results}

Hydride generation as a sample introduction technique was combined with different sensitive spectrometric detectors to measure the concentration of As species. A great majority of papers cited in the present review are devoted to its application in combination with AAS [3,6,7,12,15,16,18-20, $24,25,27,33-36,38,41,46-48,50,59-62,68,70,72,75-79]$ or AFS $[2,5,9,10,13,14,17,21,22,26,28-30,32,39,40,42$, $49,51-53,55-58,63,80-82]$, due to their lower costs in comparison with other techniques, particularly the most expensive ICP-MS, and higher availability in most laboratories. Occasionally, to improve sensitivity and extend the calibration range, ICP-OES is recommended $[8,37,45,71,83,84]$. Despite the intrinsic advantages of ICP-MS like extremely high sensitivity and a wide dynamic range, i.e., adequate for determination of (ultra)trace quantities of As, this method is rarely applied [11,31,44,53,54].

Generally, the quantification of As was carried out using external calibration with simple standards (e.g., $[6,12,15,20,27-29,32-36,49,80,83]$ ) or procedural blank-based standard (matrix-matched) solutions 
(e.g., $[2,28,38,44-47,53,54,59,77])$ prepared in the same way as samples (including pre-reduction) to keep the same acidification and the effect of additional reagents used in HG for As species (matrix-matched standards). To account for potential matrix-interfering effects from associated sample constituents, calibration by standard addition was also performed $[6,8,17,27,33,34,36,59,72]$. The lack of statistically significant differences between the slopes of these calibration curves indicated that there were no interferences, so simple external calibration was acceptable for analysis [6,27,33,34,36,59]. Frequently, to avoid or minimize intensive foam and bubble formation in the gas-liquid separator during HG, usually affecting the As response, silicone-based antifoam emulsions or alcohol-based agents (e.g., n-octanol) were added to the reductant or sample solutions $[18,23,27,28,30,40,44-46,49,50,53,54,59]$.

It should be noted that detailed verification of the analytical performance of each method was made by evaluating several quality criteria, including accuracy, (procedural) blanks and analyte-specific figures of merit such as precision (as relative standard deviation, \%RSD), LODs and linearity ranges of calibration curves.

The most reliable approach to demonstrate the accuracy of the whole method of As speciation was based on the analysis of certified reference materials (CRMs). Unfortunately, except for few such CRMs, e.g., NIST 2669 (Human Urine) [4], ERM-BC211 (Rice Flour) [38,46-48] and NIST 1568b (Rice Flour) [43], which were developed for As speciation, commercially available CRMs of different matrices with provided certified values of various As species are scare. Nevertheless, other CRMs could be used for As speciation. In this case, the content of As species was analyzed, and then the sum of their concentrations was compared with the certified value of the total As content $[10,38,39,44,50-52,81]$. Similarly, the content of As species in a real sample could also be analyzed, and then the sum of their concentrations could be compared with the total As content obtained after sample decomposition by wet digestion $[2,7,40,44,48,49,51]$. Due to a lack of proper CRMs, the reliability of the proposed methods was demonstrated by a spike-and-recovery test, based on the addition of known amounts of As species and the application of the whole procedure. Another way to check the accuracy of a newly developed methods is to compare their results with those obtained using well-established methods, e.g., HG-AAS with HPLC-HG-AFS [72], HPLC-ICP-MS [46,79], LC-ICP-MS [7], ETAAS [27] or HG-AFS [59]; HG-AFS with ICP-MS [2,49,50], IC-HG-AFS [28] or HPLC-HG-AFS [5]; HG-ICP-MS with HPLC-(HG)-ICP-MS [44,53,54] or HPLC-HG-AFS [53]. Two different sample preparation procedures, i.e., the reference and the one being examined, followed by measurements using the same detection technique, can also be applied for this purpose, i.e., a slurry sampling versus complete acid digestion in a digest block with a cold finger followed by HG-AAS [33,34], or in a microwave oven (microwave-assisted digestion) followed by ICP-MS [49].

\section{Samples and Their Preparation}

The usefulness of the HG technique for non-chromatographic speciation analysis of As is reflected in a wide spectrum of analyzed samples. This included various environmental, food and biological/clinical materials as follows:

- $\quad$ natural/environmental waters, i.e., mainly drinking $[2-5,14,18,71,83]$, tap $[4,9,15-21,84,85]$, ground $[4,6-14,83]$, underground $[3,15,16]$, sea $[3,10,11,17,24,25,68,80]$, lake $[10,14,20-24,80]$, river $[10,14,20-22,75,82,86]$, waste $[4,21]$ and snow and rain [26]

- $\quad$ sediments [72,75-77]

- $\quad$ soil $[32,76,77]$

- $\quad$ ash [72]

- $\quad$ phosphate rocks [33]

- $\quad$ airborne particular matter [34]

- $\quad$ plants [75]

- $\quad$ agricultural agents, i.e., phosphate fertilizers [33,36], herbicides [37] and pesticides [37]

- glass [35] 
- beverages, i.e., alcoholic (wine [27,28]) and alcohol-free (fruit juices [30] and tea [29]),

- cereals, i.e., rice and/or rice products [22,38,41-48] and semolina [39],

- milk [49]

- mushrooms [50]

- $\quad$ vegetables, i.e., eggplant [51], chard [51]

- marine organisms, i.e., seafood [44,52,53,79,81], seaweed/algae [44,53-55] and plankton [70]

- pharmaceuticals, i.e., Chinese medicines [56-58,78], dietary supplements [59]

- biological fluids/tissue, i.e., human urine [4,62], serum [4], cells [60,61,63] and blood and blood plasma [31]

Unfortunately, to determine the concentrations of particular As species by atomic and/or mass detection methods along with the HG technique, samples need to be in a liquid form. When focusing on speciation analyses, special attention should be paid to the sample preparation step in order to maintain the original characteristics of the species and avoid any changes in a species distribution.

Sample preparation is unnecessary when analyzing simple liquid samples like water. Usually, before analysis, natural/environmental water samples were only filtered $[9,13]$ through $0.22[26]$ or $0.45 \mu \mathrm{m}$ membrane filters [4,7,10,11,14,16-21,23-25], or centrifuged [84] to remove suspended solids or insoluble materials. They could be also acidified to 0.01 [CP2] or $0.1 \mathrm{~mol} \mathrm{~L}^{-1} \mathrm{HCl}$ [3] or $0.1-1 \% \mathrm{HNO}_{3}[11,16]$ before $[16,23]$ or after [11] filtering. In some cases, waters were stabilized with concentrated $\mathrm{HNO}_{3}$ [6] or $\mathrm{HCl}$ [12] immediately after collection. However, the addition of an oxidizing acid would likely lead to erroneous results due to transformations between $\mathrm{As}(\mathrm{III})$ and $\mathrm{As}(\mathrm{V})$ forms [6], or an interfering effect in the determination of DMA [68]. Occasionally, EDTA [15] or NaF [80] were added to mask interferences in As determination; if precipitation took place afterward, samples were filtered [21]. Samples could also be preserved during storage with a high concentration of an Fe(II) salt, added to slow down the oxidation process of $\mathrm{As}(\mathrm{III})$ to $\mathrm{As}(\mathrm{V})$ in the presence of microbial activity or oxidizing substances like Fe(III) or Mn(IV) [12,83]. Interestingly, by accomplishing HG with a pervaporation technique used prior to the detection of As species, aqueous "dirty" samples (with a suspended particulate matter) could be analyzed as received, i.e., without any previous filtration $[82,86]$. Similarly, when detection was performed with the HG technique (not PN), simple cleaning comprising filtration with a $0.45 \mu \mathrm{m}$ filter was sufficient to yield accurate results for fruit juices [30]. For more complex matrices such as alcoholic beverages, a minimal pre-treatment was required to overcome potential interference from matrix components. However, $20 \%$ evaporation of the sample volume [27] or only 5- to 10-fold dilution [28] were enough to completely remove ethanol and get rid of interference during analyses of wines by HG-AAS/AFS. Similarly, liquid rice-based products (wine, beer, vinegar) could simply be diluted (2- to 5-fold) with $0.28 \mathrm{~mol} \mathrm{~L}^{-1} \mathrm{HNO}_{3}$ prior to determination of As(III,V) by HG-AAS [47]. A direct dilution pre-treatment with water was found to be adequate to prepare biological/clinical samples such as serum, urine and blood prior to spectrometric measurements combined with HG [4,31,60-63].

In contrast to liquid samples, As speciation in solid matrices (e.g., food and environmental samples) is a challenge, since all As species must be firstly isolated from the sample matrix before further separation and detection. In contrast, to determine the total As content (usually preceded by wet digestion with aggressive reagents, i.e., commonly concentrated acids), mild but very efficient extraction is required for speciation purposes, ensuring a complete release of As compounds to be determined, albeit with no change in the original identity and concentrations of the individual As species, i.e., oxidation of $\mathrm{As}(\mathrm{III})$ to $\mathrm{As}(\mathrm{V})$ form and degradation of o-As to i-As forms. Frequently, this was achieved by solvent extraction (LE) with water [70], diluted solutions of $\mathrm{HNO}_{3}$ [36,38,40,43,46,52], $\mathrm{H}_{3} \mathrm{PO}_{4}[39,50,51]$ and $\mathrm{HCl}[33,34,75]$ or a water:methanol mixture [81]. On the other hand, solvents with partial conversion between As species could also be used. They led to the solubilization of As species, followed by the oxidation of As(III) to As(V), but without decomposition of organic As species to $\mathrm{As}(\mathrm{V})$. Typically, extraction and oxidation were carried out in the presence of $\mathrm{H}_{2} \mathrm{O}_{2}$, which was most 
often used to facilitate the extraction of total i-As species from samples in the form of As(V) $[44,53,54]$. Agua regia was also found to be effective in this regard [45]. In general, extractions were carried out at elevated temperatures $\left(<100^{\circ} \mathrm{C}\right)$; hence, heating was applied from hot-plates [38,47], shaking water baths (WBs) [41,43] or microwave (MW)-assisted radiation. Nevertheless, MW-assisted extraction (MAE) using conventional MW systems was preferred [38,44,46,53,54,81] to accelerate the release of the analyte into solutions. For As speciation, several procedures were developed based on the application of classical LE. To selectively determine As(III,V) and i-As in rice and rice products by HG-AAS or HG-AFS, extraction with $0.02-0.28 \mathrm{~mol} \mathrm{~L}^{-1} \mathrm{HNO}_{3}$ by a WB $\left(90^{\circ} \mathrm{C}, 60 \mathrm{~min}\right)$ [43], under heating in a WB on a hot plate at $95^{\circ} \mathrm{C}$ for $90 \mathrm{~min}[38,47]$ or MAE $\left(95^{\circ} \mathrm{C}\right.$ for $30 \mathrm{~min}$ ) [46] was found to be advantageous. Furthermore, in one work [38], the same results as for conventional heating with $0.28 \mathrm{~mol} \mathrm{~L}^{-1} \mathrm{HNO}_{3}$ were obtained using $\mathrm{MAE}\left(\mathrm{T}_{\max } 95^{\circ} \mathrm{C}, 40 \mathrm{~min}\right)$ with $0.14 \mathrm{~mol} \mathrm{~L}-1 \mathrm{HNO}_{3}$. Otherwise, $\mathrm{MAE}\left(50{ }^{\circ} \mathrm{C}\right.$, $5 \mathrm{~min})$ in a water:methanol $(1: 4, \mathrm{v}: \mathrm{v})$ mixture was the procedure of choice to quantitatively extract all four As species from fish prior to HG-AAS measurements [81]. In cases of selective determination of i-As as As(V) in rice and samples of marine origin (seafood, seaweed), HG-ICP-MS, HG-AAS or HG-AAS, MAE (85-95 ${ }^{\circ} \mathrm{C}$ for 20-40 $\left.\mathrm{min}\right)$ [42,44,53,54,79] or WB extraction $\left(90{ }^{\circ} \mathrm{C}, 60 \mathrm{~min}\right)$ [41] with diluted $\mathrm{HNO}_{3}-\mathrm{H}_{2} \mathrm{O}_{2}$ solutions (0.06-0.1 mol L ${ }^{-1}, 1-3 \%$ or $1-2 \% \mathrm{HNO}_{3}$ combined with $3 \% \mathrm{H}_{2} \mathrm{O}_{2}$ ) were selected. In the case of the extraction procedure with water as the solvent, it was demonstrated that As(III) and As(V) were stable during MAE (800 W, $10 \mathrm{~min})$ of plankton samples, and microwave heating was not able to reduce pentavalent inorganic As [70].

More recently, solvent extraction procedures supported by ultrasonic (US) agitation at room temperature (RT), realized using ultrasound WBs [36,39,40,45,50-52,75], have been recommended for the speciation of As. However, sample sonication at elevated temperature $\left(80^{\circ} \mathrm{C}\right)$ has also been practiced [40]. Accordingly, regarding individual speciation, a simple procedure for the determination of As(III) and i-As in phosphate fertilizers by HG-AAS was described by Rezende et al. [36], in which samples were sonicated for $35 \mathrm{~min}$ with $0.35 \%$ Triton X-114 and $6.5 \mathrm{~mol} \mathrm{~L}{ }^{-1} \mathrm{HNO}_{3}$. In another work [75], for As speciation in environmental samples (sediment, plant) by $\mathrm{HG}$-AFS, US using $6.0 \mathrm{~mol} \mathrm{~L}^{-1} \mathrm{HCl}^{-}$ assured the quantitative extraction of $\mathrm{As}(\mathrm{III}), \mathrm{As}(\mathrm{V})$ and DMA; only $10 \mathrm{~min}$ was required for sample preparation. Fast US-assisted extraction (10 min) with $1 \mathrm{~mol} \mathrm{~L}{ }^{-1} \mathrm{H}_{3} \mathrm{PO}_{4}-0.1 \%$ Triton XT-100 and of $0.1 \%$ EDTA (as a surface cleaning reagent) was also proposed by Gonzalvez et al. [50] to determine As(III) and As(V) by HG-AFS in cultivated and wild mushroom samples of different origins. Similarly, four As species (As(III,V), DMA, MMA) were selectively extracted during 10-20 min sonication of fish [52], cereals [39] and vegetables [51] samples in a mixture of 3 mol L-1 $\mathrm{HNO}_{3}$ [52] or $1 \mathrm{~mol} \mathrm{~L}^{-1}$ $\mathrm{H}_{3} \mathrm{PO}_{4}[39,51]$ and $0.1 \%$ Triton-X114, combined with $0.1 \%$ EDTA and analyzed by HG-AFS. Finally, Wang et al. [40] extracted these four target As species from rice by sonication at $80{ }^{\circ} \mathrm{C}$ for $10 \mathrm{~min}$ with $1 \% \mathrm{HNO}_{3}$; the whole procedure was repeated three times. US extraction was also advantageous for operational As speciation. Sonication of samples with aqua regia for $15 \mathrm{~min}$ was used to release four As species from the rice sample matrix, leading to oxidation of As(III) into As(V), but without demethylation of DMA and MMA [45]. i-As species were then determined by HG-ICP-OES.

A promising excellent alternative to traditional extraction is slurry sampling (SS) based on direct analysis of solid particles of sample dispersed in a liquid phase (mainly diluted acids). Furthermore, it was demonstrated that this sample preparation made it possible to speciate i-As, whereas individual species of As did not undergo any changes in oxidation state. In view of this, recently, some analytical approaches to As speciation in various solid matrices, i.e., airborne particular matter [34], phosphate fertilizers and rocks [33], milk [49] and dietary supplements [59], have been developed, adopting the SS and HG techniques and atomic spectrometry detection. By contrast to traditional LE, Macedo et al. [33,34] employed SS with HG-AAS to determine the total i-As and As(III) in various environmental materials including phosphate fertilizers and rocks [33], and airborne particulate matter samples (filters) [34]. Under optimal conditions, sample portions were mixed with $4.0-6.0 \mathrm{~mol} \mathrm{~L}^{-1} \mathrm{HCl}$ and sonicated at RT for $30 \mathrm{~min}$. The resulting sample slurries were diluted with deionized water and further analyzed. Additionally, the bioavailability of As from a breathable particulate matter, which had entered lung 
fluids, was investigated [34]. In this scenario, deionized water instead of $\mathrm{HCl}$ was used as the extracting medium, following the same extraction and quantification methods for the As content. Cava-Montesinos et al. [49] developed a sensitive procedure for the determination of As(III) and As(V) in milk samples by HG-AFS. It was based on the leaching of As species from milk through sonication with aqua regia at RT for $10 \mathrm{~min}$, followed by dilution with $\mathrm{HCl}$. Importantly, it was demonstrated that neither As(III) nor As(V) were modified by the proposed sample treatment. This was in contrast to $[39,45]$, where it was reported that the integrity of As(III) and As(V) in aqua regia was not preserved, leading to the oxidation of $\mathrm{As}(\mathrm{III})$ to $\mathrm{As}(\mathrm{V})$ during As species extraction from cereals such as rice $[39,45]$ and wheat semolina [39]. The speciation of inorganic As in various dietary supplements (tablets, capsules) by using the SS and HG-AAS was described by Sun et al. [59], wherein As(III) and As(V) were isolated from the sample matrix during heating with $50 \% \mathrm{HCl}$ in a WB for boiling for 5-10 min. Since adequate sample slurry preparation is of major importance in this sample technique, critical studies, aiming to achieve stability and uniformity of slurries, were undertaken. The best results were obtained for a sample particle size of $54 \mu \mathrm{m}$ and a $0.3 \%$ agar reagent added to increase the viscosity of the medium. To achieve adequate homogeneity, ultrasonic agitation in an ultrasonic bath at $350 \mathrm{~W}$ for 30 min was applied.

Specific extraction protocols for the determination of inorganic As in ash, sediment and soil were also proposed [32,72,77]. Accordingly, Gonzalez et al. [72] employed a two-step, sequential extraction procedure using water and a $1.0 \mathrm{mmol} \mathrm{L}^{-1}$ phosphate buffer to identify As oxidation states in soluble and exchangeable As fractions, respectively, in fly ash and sediment samples. Similarly, Shi et al. [32] introduced a four-step extraction procedure, where deionized water, $0.6 \mathrm{~mol} \mathrm{~L}-1 \mathrm{KH}_{2} \mathrm{PO}_{4}$, $1 \% \mathrm{HCl}$ and $1 \% \mathrm{NaOH}$ were sequentially used to leach extractable As forms from soils. Solubilized inorganic As compounds were speciated in these extracts by HG-AAS [72] and HG-AFS [32]. Shortened procedures were also proposed to assess the potential bioavailability of toxic As species. Accordingly, Petrov et al. [77] selectively extracted all four As species with EDTA $\left(0.05 \mathrm{~mol} \mathrm{~L}^{-1}\right.$, $\left.\mathrm{pH} 6-7\right)$ from soils and sediments prior to analysis of the EDTA-extractable As fraction by HG-AAS on the content of the sum of As(III,V), DMA and MMA. In another two works [56,57], to understand the solubility, mobility and transport of As in herbaceous plant samples for traditional Chinese medicines (TCMs), as well as commercially available TCMs (herbs), extraction with low concentration $\mathrm{HCl}$ [57] and water [56] was recommended. In cases of plants, powdered samples were extracted overnight (RT) with 1\% $\mathrm{HCl}$, then filtered and diluted with water. To investigate leachable As species from commercial TCMs, samples were placed in water, heated on a hot-plate to boil for $30 \mathrm{~min}$, and then cooled and filtered. Concentrations of As(III) and total i-As in the obtained sample solutions were measured directly by HG-AFS. Because water is an attractive extracting solution for As, simple brewing, i.e., an everyday culinary process of tea infusion preparation, was also proposed for the speciation of As in tea [29]. For this purpose, a portion of tea was extracted with water for $20 \mathrm{~min}$ at $100{ }^{\circ} \mathrm{C}$. Next, a sample suspension was filtered, diluted with water and analyzed for its As(III,V) content by HG-AFS.

Adequate extraction procedures are crucial for speciation analyses of specific solid samples too. Thus, critical studies were performed by do Nascimento et al. [35] for speciation analysis of As in glass. The authors compared four procedures for glass decomposition in order to determine inorganic As species in various commercial clear, green or amber glass ampoules, bottles and containers. Alkaline fusion with $\mathrm{Na}_{2} \mathrm{CO}_{3}\left(2 \mathrm{~h}\right.$ at $\left.900{ }^{\circ} \mathrm{C}\right)$ and glass dissolution in $40 \% \mathrm{NaOH}$ or diluted $\mathrm{HF}(24 \%)$ for $48 \mathrm{~h}$ with or without microwave irradiation (10-50 min at $174 \mathrm{~W}$ ) were tested. Problems related to either losses of As or stability of As(III) and As(V) forms, as well as incomplete real sample dissolution, were observed using alkaline fusion and the treatment with $\mathrm{NaOH} / \mathrm{HF}$ supported by heating. Satisfactorily, a cold diluted HF-based strategy was the procedure of choice, and complete recoveries of both As species were obtained. 


\section{Non-Chromatographic As Speciation by Selective HG}

The separation of As species in (prepared) sample solutions or sample extracts by selective HG and subsequent spectrometric detection is the most widely applied strategy for non-chromatographic As speciation, combined with the HG technique. Generally, using HG, different reactivities of As(III), As(V), DMA and MMA at various HG reaction conditions are used for the selective generation of individual hydrides. These As hydrides can be generated either selectively at different chemical conditions, or together with other species in various reaction media. Despite these essential advantages, As speciation by HG is focused mainly on i-As species and procedures discriminating between As(III) and As(V) $[2,6,12,15,17,20,27,29,32-36,38,47,49,50,56,57,59,72,80,83]$ or ensuring the selective determination of i-As (as As(III)) [44-46,53,54] are those dominating.

\subsection{Case of Inorganic Arsenic (As(III) and As(V))}

\subsubsection{Individual Speciation, i.e., Separately Determined $\mathrm{As}(\mathrm{III})$ and $\mathrm{As}(\mathrm{V})$}

Several approaches provided by selective HG can be used for the differentiation of $\mathrm{As}(\mathrm{III})$ and $\mathrm{As}(\mathrm{V})$. Accordingly, species selective HG from As(III,V) is based on:

(a) Different reaction media, i.e., the acidity-dependent reduction reaction between As species and $\mathrm{NaBH}_{4}$ to generate hydrides (affected strongly by the type and the concentration of acid or buffer solutions used, as well as the $\mathrm{pH}$ of the reacting medium).

(b) The reaction rate with $\mathrm{NaBH}_{4}$, i.e., differences in the reduction efficiency between $\mathrm{As}(\mathrm{III})$ and $\mathrm{As}(\mathrm{V})$ at different $\mathrm{NaBH}_{4}$ concentrations (especially low) in the acid medium.

(c) The absence or presence of additives like pre-reduction agents or other specific organic substances including chelating and masking reagents. The addition of various pre-reductants ensures that any $\mathrm{As}(\mathrm{V})$ is in the $\mathrm{As}(\mathrm{III})$ form before the reaction with $\mathrm{NaBH}_{4}$ takes place; this provides the highest sensitivity in the HG technique for As, and hence, makes it possible to quantify the total As content.

Considering the approaches mentioned above, the determination of $\mathrm{As}(\mathrm{III})$ and $\mathrm{As}(\mathrm{V})$ is quite easy. Generally, a three-step scheme is needed, comprising: (i) the selective determination of As(III) in the presence of $\mathrm{As}(\mathrm{V})$; (ii) the determination of total i-As as As(III) after a pre-reduction step for $\mathrm{As}(\mathrm{V})$ with a suitable pre-reductant; and finally, (iii) the calculation of the $\mathrm{As}(\mathrm{V})$ content as the difference $[\mathrm{As}(\mathrm{V})=\mathrm{i}-\mathrm{As}-\mathrm{As}(\mathrm{III})]$. Accordingly, different reaction media (acids, buffers) $[2,6,29,32-36,70,72]$ and variable concentrations of $\mathrm{NaBH}_{4}$ in both soft and highly acidic conditions $[12,15,20,27,38,47,72,80,83]$ were used for the selective determination of As(III) and total i-As. Concurrently to the subtracting method, since responses of $\mathrm{As}(\mathrm{III})$ and $\mathrm{As}(\mathrm{V})$ in different $\mathrm{HG}$ reaction conditions are not the same, linear independent equations relating their analytical signals versus concentrations were also used for quantification $[17,29,49,50,56]$. The optimum conditions for inorganic As speciation (individual) in various matrices by selective HG and atomic spectrometry detection with LODs of $\leq 1 \mu \mathrm{g} \mathrm{L}{ }^{-1}$ are summarized in Table 2. 
Table 2. Non-chromatographic procedures for individual speciation, i.e., selective determination of $\operatorname{As}(\mathrm{III})$ and $\mathrm{As}(\mathrm{V})$.

\begin{tabular}{|c|c|c|c|c|c|c|}
\hline Matrix & Species & Sample Preparation ${ }^{a}$ & Speciation Approach & Detection & $\mathrm{LOD}^{\mathrm{b}}, \mu \mathrm{g} \mathrm{L}^{-1}$ & Ref \\
\hline \multicolumn{7}{|c|}{ Redox Property of $\mathrm{NaBH}_{4}$ (kinetic-dependent reduction reaction between $\mathrm{NaBH}_{4}$ and $\mathrm{As}(\mathrm{IIII}, \mathrm{V})$ forms) } \\
\hline natural waters & $\begin{array}{l}\mathrm{As}(\mathrm{III}) \\
\mathrm{As}(\mathrm{V})\end{array}$ & direct analysis & $\begin{array}{c}\text { I. Selective As(III): } \\
\text { S: not acidified, A: } 10 \mathrm{M} \mathrm{HCl}, \mathrm{R}: 0.05 \% \mathrm{NaBH}_{4} \\
\text { II. } i \text {-As (as As(III)): } \\
\text { S: L-cysteine, A: } 10 \mathrm{M} \mathrm{HCl}, 0.6 \% \mathrm{NaBH}_{4}\end{array}$ & HG-ICP-OES & 1.0 & {$[83$} \\
\hline $\begin{array}{c}\text { water CRMs } \\
\text { (TMDA-54.3, CASS-4) }\end{array}$ & $\begin{array}{l}\text { As(III) } \\
\text { As(V) }\end{array}$ & direct analysis & $\begin{array}{c}\text { S: } 4 \mathrm{M} \mathrm{HCl}, \mathrm{A}: 6 \mathrm{M} \mathrm{HCl}, 0.2 \% \mathrm{NaBH}_{4} \\
\text { II. } i \text {-As (as As(III)): }\end{array}$ & HG-AFS & 0.05 & {$[80]$} \\
\hline natural waters & $\begin{array}{l}\text { As(III) } \\
\text { As(V) }\end{array}$ & direct analysis & $\begin{array}{c}\text { S: KI-ascorbic acid-4 M HCl, A: } 6 \mathrm{M} \mathrm{HCl}, 0.2 \% \mathrm{NaBH}_{4} \\
\text { I. Selective As(III): } \\
\text { S: } 0.1 \mathrm{M} \mathrm{HCl}, 4.0 \% \mathrm{NaBH}_{4} \\
\text { II. i-As (as As(III)): } \\
\text { S: KI-ascorbic acid-1 } \mathrm{M} \mathrm{HCl}, 4.0 \% \mathrm{NaBH}_{4} \\
\text { I. Selective As(III): }\end{array}$ & HG-AAS & 1.2 & {$[15]$} \\
\hline natural waters & $\begin{array}{l}\mathrm{As}(\mathrm{III}) \\
\mathrm{As}(\mathrm{V})\end{array}$ & direct analysis & $\begin{array}{c}\text { A: } 1.5 \mathrm{M} \mathrm{HCl}, \mathrm{R}: 0.5 \% \mathrm{NaBH}_{4} \\
\text { II. } i \text {-As }(\text { as } A s(I I I)): \\
\text { A: } 9.0 \mathrm{M} \mathrm{HCl} 3.0 \% \mathrm{NaBH}_{4}\end{array}$ & HG-AAS & $\begin{array}{l}\text { As(III): } 0.1 \\
\text { As(V): } 0.06\end{array}$ & {$[20]$} \\
\hline ground water & $\begin{array}{l}\text { As(III) } \\
\text { As(V) }\end{array}$ & direct analysis & $\begin{array}{c}\text { I. Selective As(III): } \\
\text { S: } 0.1 \mathrm{M} \mathrm{HCl}, \mathrm{A}: 2 \mathrm{M} \mathrm{HCl}, 0.035 \% \mathrm{NaBH}_{4}, \\
\text { II. } \text { i-As (as As(III)): } \\
\text { S: KI-ascorbic acid-2.6 M HCl, A: } 2 \mathrm{M} \mathrm{HCl}, \mathrm{R}: 0.2 \% \mathrm{NaBH}_{4} \text {, } \\
\text { I. Selective As(III): }\end{array}$ & HG-AAS & $\begin{array}{c}\mathrm{As}(\mathrm{III}): 1.4 \\
\mathrm{As}(\mathrm{V}): 0.6\end{array}$ & {$[12]$} \\
\hline rice & $\begin{array}{l}\mathrm{As}(\mathrm{III}) \\
\mathrm{As}(\mathrm{V})\end{array}$ & MAE (0.14 $\left.\mathrm{M} \mathrm{HNO}_{3}\right)$ & $\begin{array}{c}\text { S: } 0.14 \mathrm{M} \mathrm{HNO}_{3} \text {, A: } 10 \mathrm{M} \mathrm{HCl}, 0.1 \% \mathrm{NaBH}_{4} \\
\text { II. } i \text {-As (as As(III)): } \\
\text { S: } 0.14 \mathrm{M} \mathrm{HNO}_{3}-\mathrm{KI} \text {-ascorbic acid-1.2 M HCl, } 10 \mathrm{M} \mathrm{HCl} \text {, R: } \\
0.1 \% \mathrm{NaBH}_{4}\end{array}$ & HG-AAS & $\begin{array}{l}\mathrm{As}(\mathrm{III}): 0.07 \\
\mathrm{As}(\mathrm{V}): 0.14\end{array}$ & {$[38$} \\
\hline rice products & $\begin{array}{l}\mathrm{As}(\mathrm{III}) \\
\mathrm{i}-\mathrm{As}\end{array}$ & $\mathrm{HP}\left(0.28 \mathrm{M} \mathrm{HNO}_{3}\right)$ & $\begin{array}{c}\text { I. Selective As(III): } \\
\text { S: } 0.28 \mathrm{M} \mathrm{HNO}_{3} \text {, A: } 10 \mathrm{M} \mathrm{HCl}, 0.1 \% \mathrm{NaBH}_{4} \\
\text { II. } \mathrm{i} \text {-As (as As(III)): } \\
\text { S: } 0.28 \mathrm{M} \mathrm{HNO}_{3} \text {-KI-ascorbic acid-1.9 M HCl, } 10 \mathrm{M} \mathrm{HCl} \text {, R: } \\
0.1 \% \mathrm{NaBH}_{4}\end{array}$ & HG-AAS & $\begin{array}{c}\text { As(III): } 0.08 \\
\text { i-As: } 0.14\end{array}$ & {$[47]$} \\
\hline
\end{tabular}


Table 2. Cont

\begin{tabular}{|c|c|c|c|c|c|c|}
\hline Matrix & Species & Sample Preparation ${ }^{a}$ & Speciation Approach & Detection & $\mathrm{LOD}^{\mathrm{b}}, \mu \mathrm{g} \mathrm{L}^{-1}$ & Ref. \\
\hline \multicolumn{7}{|c|}{ Specific HG from $\mathrm{As}(\mathrm{III})$ and $\mathrm{As}(\mathrm{V})$ under controlled $\mathrm{pH}$ conditions or different reaction media } \\
\hline drinking water & $\begin{array}{l}\mathrm{As}(\mathrm{III}) \\
\mathrm{As}(\mathrm{V})\end{array}$ & direct analysis & $\begin{array}{c}\text { I. Selective As(III): } \\
\text { S: Tris-HCl buffer (pH 7.2), R: } 3.0 \% \mathrm{NaBH}_{4} \\
\text { II. } \mathrm{i}-\text { As (as As(III)): } \\
\text { S: Tris-HCl (pH 7.2)-TGA, R: } 3.0 \% \mathrm{NaBH}_{4} \\
\text { I. Selective As(III): }\end{array}$ & HG-AFS & $\begin{array}{l}\mathrm{As}(\mathrm{III}): 0.027 \\
\mathrm{As}(\mathrm{V}): 0.036\end{array}$ & [2] \\
\hline ground water & $\begin{array}{l}\text { As(III) } \\
\text { As(V) }\end{array}$ & direct analysis & $\begin{array}{c}\text { S: not acidified, A: } 0.1 \mathrm{M} \text { citric acid, } \mathrm{R}: 0.6 \% \mathrm{NaBH}_{4} \\
\text { II. i-As (as As(III)): } \\
\text { S: KI-6.5 M HCl, A: } 10 \mathrm{M} \mathrm{HCl}, \mathrm{R}: 0.6 \% \mathrm{NaBH}_{4}\end{array}$ & HG-AAS & 0.4 & [6] \\
\hline $\begin{array}{l}\text { ash and soil CRMs } \\
\text { (NIST 1633b } \\
\text { GBW07302 } \\
\text { GBW07311) }\end{array}$ & $\begin{array}{l}\mathrm{As}(\mathrm{III}) \\
\mathrm{As}(\mathrm{V})\end{array}$ & $\begin{array}{l}\text { two-step sequential LE } \\
\left(\mathrm{H}_{2} \mathrm{O}, 1 \mathrm{mM}\right. \\
\text { phosphate buffer })\end{array}$ & $\begin{array}{c}\text { I. Selective As(III): } \\
\text { S: citric buffer (pH 4.5), A: } 10 \% \mathrm{HCl}, \mathrm{R}: 0.3 \% \mathrm{NaBH}_{4} \\
\text { optionally: S: } 2 \% \mathrm{HCl}, \mathrm{A}: 2 \% \mathrm{HCl}, \mathrm{R}: 0.3 \% \mathrm{NaBH}_{4} \\
\text { II. } i \text {-As (as As(III)): } \\
\text { S: KI-ascorbic acid-HCl, A: } 10 \% \mathrm{HCl}, \mathrm{R}: 0.3 \% \mathrm{NaBH}_{4} \\
\text { optionally: S: } 0.46 \% \text { TGA in sample, A: } 10 \% \mathrm{HCl} \\
\text { R: } 0.3 \% \mathrm{NaBH}_{4}\end{array}$ & HG-AAS & $\begin{array}{l}\text { As(III): } 0.07 \\
\text { As(V): } 0.06\end{array}$ & [72] \\
\hline soil & $\begin{array}{l}\operatorname{As}(\mathrm{III}) \\
\operatorname{As}(\mathrm{V})\end{array}$ & $\begin{array}{l}\text { four-step sequential LE } \\
\left(\mathrm{H}_{2} \mathrm{O}, 0.6 \mathrm{M} \mathrm{KH} \mathrm{PO}_{4}\right. \\
1 \% \mathrm{HCl}, 1 \% \mathrm{NaOH})\end{array}$ & $\begin{array}{c}\text { I. Selective As(III): } \\
\text { S: } 0.1 \mathrm{M} \text { citric acid, R: } 1.0 \% \mathrm{NaBH}_{4} \\
\text { II. } i \text {-As (as As(III)) } \\
\text { S: } 0.1 \mathrm{M} \text { citric acid-L-cysteine, } 1.0 \% \mathrm{NaBH}_{4} \\
\text { I. Selective As(III): }\end{array}$ & HG-AFS & $\begin{array}{l}\text { As(III): } 0.11 \\
\text { As(V): } 0.07\end{array}$ & [32] \\
\hline glass & $\begin{array}{l}\mathrm{As}(\mathrm{III}) \\
\mathrm{As}(\mathrm{V})\end{array}$ & dissolution $(24 \% \mathrm{HF})$ & $\begin{array}{c}\text { S: citric buffer (pH 4.5), R: } 1.0 \% \mathrm{NaBH}_{4} \\
\text { II. i-As (as As(III)): } \\
\text { S: } 6.0 \mathrm{M} \mathrm{HCl,} \mathrm{R:} 1.0 \% \mathrm{NaBH}_{4} \\
\text { I. Selective As(III): }\end{array}$ & HG-AAS & & [35] \\
\hline $\begin{array}{l}\text { airborne particulate } \\
\text { matter }\end{array}$ & $\begin{array}{l}\text { As(III) } \\
\text { i-As }\end{array}$ & $\mathrm{SS}(4 \mathrm{M} \mathrm{HCl})$ & $\begin{array}{c}\text { S: citric buffer (pH 7.1), A: } 2 \mathrm{M} \mathrm{HCl}, \mathrm{R}: 2.15 \% \mathrm{NaBH}_{4} \\
\text { II. } \mathrm{i} \text {-As (as As(III)): } \\
\text { S: KI-ascorbic acid-1.8 M HCl, A: } 2 \mathrm{M} \mathrm{HCl,} \mathrm{R:} 2.15 \% \mathrm{NaBH}_{4} \\
\text { I. Selective As(III): }\end{array}$ & HG-AAS & 0.1 & {$[34]$} \\
\hline $\begin{array}{l}\text { phosphate fertilizer } \\
\text { and rock }\end{array}$ & $\begin{array}{c}\mathrm{As}(\mathrm{III}) \\
\mathrm{i}-\mathrm{As}\end{array}$ & $\mathrm{SS}(6 \mathrm{M} \mathrm{HCl})$ & $\begin{array}{c}\text { S: citric buffer (pH 7.1), A: } 2 \mathrm{M} \mathrm{HCl}, \mathrm{R}: 2.15 \% \mathrm{NaBH}_{4} \\
\text { II. } i-A s(\text { as } A s(I I I)) \\
\text { S: KI-ascorbic acid-2 M HCl, A: } 2 \mathrm{M} \mathrm{HCl,} \mathrm{R:} 2.15 \% \mathrm{NaBH}_{4}\end{array}$ & HG-AAS & 0.1 & [33] \\
\hline
\end{tabular}


Table 2. Cont

\begin{tabular}{|c|c|c|c|c|c|c|}
\hline Matrix & Species & Sample Preparation ${ }^{a}$ & Speciation Approach & Detection & $\mathrm{LOD}^{b}, \mu g \mathrm{~L}^{-1}$ & Ref. \\
\hline phosphate fertilizer & $\begin{array}{l}\text { As(III) } \\
\text { i-As }\end{array}$ & $\begin{array}{l}\text { US }(0.35 \% \text { Triton X-114 } \\
\left.\quad+6.5 \mathrm{M} \mathrm{HNO}_{3}\right)\end{array}$ & $\begin{array}{c}\text { I. Selective As(III): } \\
\text { S: citric buffer (pH 4.5), A: } 10 \% \mathrm{HCl}, \mathrm{R}: 0.4 \% \mathrm{NaBH}_{4} \\
\text { II. i-As (as As(III)): } \\
\text { S: thiourea, A: } 10 \% \mathrm{HCl}, \mathrm{R}: 0.4 \% \mathrm{NaBH}_{4} \\
\text { I. Selective As(III): }\end{array}$ & HG-AAS & $\begin{array}{l}\text { As(III): } 0.029 \\
\text { i-As: } 0.022\end{array}$ & [36] \\
\hline wine & $\begin{array}{l}\text { As(III) } \\
\text { i-As }\end{array}$ & EtOH evaporation & $\begin{array}{c}\text { S: } 8 \mathrm{M} \mathrm{HCl}, \mathrm{A}: 9 \mathrm{M} \mathrm{HCl}, \mathrm{R}: 0.2 \% \mathrm{NaBH}_{4} \\
\text { II. } i \text {-As }(\text { as As(III)): } \\
\text { S: } \mathrm{KI}-8 \mathrm{M} \mathrm{HCl}, \mathrm{A}: 9 \mathrm{M} \mathrm{HCl}, \mathrm{R}: 0.2 \% \mathrm{NaBH}_{4}\end{array}$ & HG-AAS & 0.1 & [27] \\
\hline plankton & $\begin{array}{l}\mathrm{As}(\mathrm{III}) \\
\mathrm{As}(\mathrm{V})\end{array}$ & $\operatorname{MAE}\left(\mathrm{H}_{2} \mathrm{O}\right)$ & $\begin{array}{c}\text { I. Selective As(III): } \\
\text { S: citric buffer (pH 4.5), A: } 2 \% \mathrm{HCl}, \mathrm{R}: 0.1 \% \mathrm{NaBH}_{4} \\
\text { II. } i \text {-As (as As(III)): } \\
\text { S: citric buffer (pH 4.5)-L-cysteine, A: } 2 \% \mathrm{HCl} \\
\text { R: } 0.1 \% \mathrm{NaBH}_{4}\end{array}$ & HG-MF-AAS & 2.0 & {$[70]$} \\
\hline \multicolumn{7}{|c|}{ Species-selective respond toward two different reducing conditions (As speciation based on systems of linear independent equations) } \\
\hline milk & $\begin{array}{l}\mathrm{As}(\mathrm{III}) \\
\mathrm{As}(\mathrm{V})\end{array}$ & SS (aqua regia) & $\begin{array}{l}1.2 \% \mathrm{NaBH}_{4}-3.5 \mathrm{M} \mathrm{HCl} \text { without and after pre-reduction with } \\
\mathrm{KI} \text {-ascorbic acid-hydroxylamine hydrochloride in } 10.8 \mathrm{M} \mathrm{HCl}\end{array}$ & HG-AAS & $\begin{array}{l}\text { As(III): } 0.0081 \\
\text { As(V): } 0.0103\end{array}$ & [49] \\
\hline mushrooms & $\begin{array}{l}\mathrm{As}(\mathrm{III}) \\
\mathrm{As}(\mathrm{V})\end{array}$ & $\begin{array}{l}\mathrm{US}\left(1 \mathrm{M} \mathrm{H}_{3} \mathrm{PO}_{4}-0.1 \%\right. \\
\text { Triton } \mathrm{X}-100+0.1 \% \\
\text { EDTA })\end{array}$ & $\begin{array}{c}0.7 \% \mathrm{NaBH}_{4}-3.5 \mathrm{M} \mathrm{HCl} \text { without and after pre-reduction } \\
\text { with } \mathrm{KI} \text {-ascorbic acid in } 9 \mathrm{M} \mathrm{HCl}\end{array}$ & HG-AFS & $6.5 \mathrm{ng} \mathrm{g}^{-1 \mathrm{c}}$ & [50] \\
\hline TCM (herbs) & $\begin{array}{l}\mathrm{As}(\mathrm{III}) \\
\mathrm{As}(\mathrm{V})\end{array}$ & $\mathrm{LE}\left(\mathrm{H}_{2} \mathrm{O}\right)$ & $\begin{array}{c}1.0 \% \mathrm{KBH}_{4}-1 \mathrm{M} \mathrm{HCl} \text { without and after pre-reduction with } \\
\mathrm{KI} \text {-thiourea in } 2 \mathrm{M} \mathrm{HCl} \\
\text { I. Selective } \mathrm{As}(\mathrm{III}) \text { : }\end{array}$ & HG-AFS & 0.0797 & [56] \\
\hline tea & $\begin{array}{l}\mathrm{As}(\mathrm{III}) \\
\mathrm{As}(\mathrm{V})\end{array}$ & brewing $\left(\mathrm{H}_{2} \mathrm{O}\right)$ & $\begin{array}{c}\text { S: } 0.1 \mathrm{M} \text { citric acid- } 5 \% \mathrm{HCl}, \mathrm{A}: 5 \% \mathrm{HCl}, \mathrm{R}: 5 \% \mathrm{KBH}_{4} \\
\text { II. } i \text {-As (as As(III)): } \\
\text { S: thiourea-ascorbic acid-5\% } \mathrm{HCl}, \mathrm{A}: 5 \% \mathrm{HCl}, \mathrm{R}: 5 \% \mathrm{KBH}_{4}\end{array}$ & HG-AFS & $\begin{array}{l}\text { As(III): } 0.0070 \\
\text { As(V): } 0.0095\end{array}$ & [29] \\
\hline natural waters & $\begin{array}{l}\mathrm{As}(\mathrm{III}) \\
\mathrm{As}(\mathrm{V})\end{array}$ & direct analysis & $\begin{array}{l}\text { I. S: } 0.7 \mathrm{M} \mathrm{HCl}, \mathrm{R}: 0.7 \% \mathrm{NaBH}_{4} \\
\text { II. S: L-cysteine-0.1 M HCl, R: } 0.7 \% \mathrm{NaBH}_{4}\end{array}$ & HG-AFS & $\begin{array}{l}\mathrm{As}(\mathrm{III}) 0.013 \\
\mathrm{As}(\mathrm{V}): 0.015\end{array}$ & [17] \\
\hline
\end{tabular}


Table 2. Cont.

\begin{tabular}{|c|c|c|c|c|c|c|}
\hline Matrix & Species & Sample Preparation ${ }^{a}$ & Speciation Approach & Detection & $\operatorname{LOD}^{b}, \mu \mathrm{g} \mathrm{L}^{-1}$ & Ref. \\
\hline \multicolumn{7}{|c|}{ Specific HG from As(III) and As(V) in the presence of masking reagents } \\
\hline herbaceous plant & $\begin{array}{l}\mathrm{As}(\mathrm{III}) \\
\mathrm{As}(\mathrm{V})\end{array}$ & $\mathrm{LE}(1 \% \mathrm{HCl})$ & $\begin{array}{c}\text { I. Selective As(III): } \\
\text { S: 8-hydroxyquinoline-10\% } \mathrm{HCl}, \mathrm{R}: 2.5 \% \mathrm{NaBH}_{4} \\
\text { II. } i \text {-As (as As(III)): } \\
\text { 8-hydroxyquinoline-KI-ascorbic acid- } 10 \% \mathrm{HCl} \text {, } \\
\text { R: } 2.5 \% \mathrm{NaBH}_{4}\end{array}$ & HG-AFS & & [57] \\
\hline dietary suplements & $\begin{array}{l}\mathrm{As}(\mathrm{III}) \\
\mathrm{As}(\mathrm{V})\end{array}$ & $\mathrm{SS}(50 \% \mathrm{HCl})$ & $\begin{array}{c}\text { I. Selective As(III): } \\
\text { S: 8-hydroxyquinoline-1\% } \mathrm{HCl}, \mathrm{R}: 1.0 \% \mathrm{KBH}_{4} \\
\text { II. } \mathrm{i} \text {-As (as As(III)): } \\
\text { S: 8-hydroxyquinoline-KI-5\% HCl, R: } 1.0 \% \mathrm{KBH}_{4}\end{array}$ & HG-AAS & $\begin{array}{c}\mathrm{As}(\mathrm{III}): 0.080 \\
\mathrm{As}(\mathrm{V}): 0.089\end{array}$ & [59] \\
\hline \multicolumn{7}{|c|}{$\begin{array}{l}\text { EtOH: ethanol. HG-AAS: hydride generation atomic absorption spectrometry. HG-AFS: hydride generation atomic fluorescence spectrometry. HG-MF-AAS: metallic furnace hydride } \\
\text { generation atomic absorption spectrometry. HG-ICP-OES: hydride generation inductively-coupled plasma optical emission spectrometry. HP: heating in water bath on hot plate } \\
\text { i-As: the inorganic tri- and pentavalent As species (As(III) and } \mathrm{As}(\mathrm{V})) \text {. LE: solvent extraction. M: mol L }{ }^{-1} \text {. MAE: microwave-assisted extraction. S: sample solution. A: additional acid } \\
\text { (carrier) solution. R: reductant solution. SS: slurry sampling. TCM: traditional Chinese medicines. TGA: thioglycolic acid. US: ultrasonication in an ultrasound water bath. WB: water bath. } \\
\text { a Detailed information about sample preparation procedures described in point } 4 .{ }^{b} \text { For As(V) or i-As as As(III) without or after previous pre-reduction step. }{ }^{c} \text { In the original sample, taking } \\
\text { into account the amount of sample and the final dilution emploved in the proposed procedure. }\end{array}$} \\
\hline
\end{tabular}


It can be concluded that, on the one hand, $\mathrm{As}(\mathrm{III})$ reacts selectively with $\mathrm{NaBH}_{4}$ in buffered media (with citrates at $\mathrm{pH} 4.5[35,36,70,72]$ and at $\mathrm{pH} 7.1[33,34]$ or Tris- $\mathrm{HCl}$ at $\mathrm{pH} 7.2$ [2]) or low concentrated organic acids such as citric acid $\left(0.1 \mathrm{~mol} \mathrm{~L}^{-1}\right.$ [6,32]) or citric acid combined with $\mathrm{HCl}\left(0.1 \mathrm{~mol} \mathrm{~L}^{-1}-5 \%\right.$ [29]). On the other hand, selective $\mathrm{AsH}_{3}$ formation for As(III) without interference from As(V) can be also achieved via the $\mathrm{HG}$ reaction at (very) low $\mathrm{NaBH}_{4}$ concentrations $(0.035-0.2 \%)[12,27,38,47,72,80,83]$, using $0.65-10 \mathrm{~mol} \mathrm{~L}^{-1} \mathrm{HCl}$ as the reaction medium. Interestingly, using a batch type $\mathrm{HG}$ system, $4 \% \mathrm{NaBH}_{4}$ combined with $0.1 \mathrm{~mol} \mathrm{~L}^{-1} \mathrm{HCl}$ was found to be optimal for direct measurements of $\mathrm{As}(\mathrm{III})$ coexisting with $\mathrm{As}(\mathrm{V})$ [15]. It should be noted that the As signal increases with the concentration of $\mathrm{NaBH}_{4}$; this effect is evident, particularly for As(V). Therefore, the use of low or even very low concentrations of $\mathrm{NaBH}_{4}$ decreases the sensitivity of As(III) determination, and hence, the method LOD of As(III) would be affected too [72]. Generally, for this approach, the selected $\mathrm{NaBH}_{4}$ concentration is a compromise between the error due to the As(V) interference and the LOD value, making it possible to reliably determine the As(III) content in examined samples [12,83]. Typically, total i-As is determined at higher $\mathrm{NaBH}_{4}$ concentrations $(0.2-3 \%)[2,6,12,29,33,34,36,72,83]$ and 2-10 mol L ${ }^{-1} \mathrm{HCl}$ for the $\mathrm{HG}$ reaction after the offline pre-reduction of As(V) to As(III) using KI with ascorbic acid [6,12,34,72], thiourea (alone [36] or in combination with ascorbic acid [29]) and L-cysteine [83]. Total i-As can be also determined under the same HG reaction conditions as for As(III) after online $[2,32,80]$ or offline $[6,15,27,38,47,70]$ pre-reduction of As(V) to As(III) with KI alone [6,27], KI-ascorbic acid [15,38,47,80], L-cysteine [32,70] or thioglycolic acid (TGA) [2].

Potassium iodide is one of the most widely used reagents in the pre-reducing step; however, it works effectively only under strong acidic conditions for sample acidification, i.e., $1-11 \mathrm{~mol} \mathrm{~L}^{-1}$ $\mathrm{HCl}$ (see e.g., [6,12,13,15,27,34,38,39,45-47,49,51,52,71,80]). Sample acidifications of 2-3 mol L-1 $\mathrm{HCl}^{-1}$ were the most common [12,13,34,39,45,46,51,52]; nevertheless, lower $\mathrm{HCl}$ concentrations in sample solutions (0.1-0.7 mol L $\left.{ }^{-1}\right)$ were also used $[3,28]$. Typically, ascorbic acid is added along with KI in order to avoid the self-oxidation of $\mathrm{I}^{-}$ions to free $\mathrm{I}_{2}$ by the $\mathrm{O}_{2}$ that is present in solutions $[12,15,46,77,80]$. Importantly, the ascorbic acid concentration has no effect on the As signals; thus, its role is primarily to stabilize the pre-reduction medium [80]. Despite this, samples need to be analyzed within $6 \mathrm{~h}$ after the addition of reagents to avoid oxidation by atmospheric $\mathrm{O}_{2}$, leading to the formation of triiodide and the eventual re-oxidation of $\mathrm{As}(\mathrm{III})$ to $\mathrm{As}(\mathrm{V})$ [46]. Optionally, to transgress $\mathrm{I}_{2}$, sample solutions treated with KI alone can be heated to boil for 5-10 min [59]. Pre-reduction with KI runs commonly for 30-60 $\min [28,33,34,38,39,44,46,50-53,59,77]$, but it can be achieved in a shorter time (i.e., 5 min) by increasing the reagent concentration [80]. By contrast, reagents with thiol (-SH) functional groups such as L-cysteine, thiourea and TGA were found to be more advantageous. Besides its pre-reduction properties, thiourea can also be used as a sensitization reagent, improving the As intensity [56]. L-cysteine pre-reduces all pentavalent As species to their trivalent oxidation state forms with similar responses at a relatively low and narrow optimum $\mathrm{HCl}$ concentration range $\left(0.01-0.1 \mathrm{~mol} \mathrm{~L}^{-1}\right)[28,71,81]$. This reagent was used also to level off the responses of different As species [28,32]. The reaction time for the complete pre-reduction at RT, i.e., 30-60 min [28,52], can be shortened to less than $60 \mathrm{~s}$ at $100{ }^{\circ} \mathrm{C}$ [52]. Interestingly, pre-reduction with TGA is fast and measurements can be made just after adding this reagents to sample solutions [2,72]. Additionally, it can be used as a pre-reductant for all four As species [2], or can be treated as a reaction medium, making it possible to achieve the same responses with $\mathrm{As}(\mathrm{III})$ and $\mathrm{As}(\mathrm{V})$ [72].

Gonzalez et al. [72] compared several speciation procedures for the selective determination of As(III) in the presence of As(V) and total i-As by HG-AAS in water-soluble and phosphate-exchangeable extracts of sediments and fly ash CRMs (NIST 1633b, GBW 07311 and GBW 07302). This included (i) $\mathrm{AsH}_{3}$ generation under soft $\mathrm{HG}$ conditions, i.e., low $\mathrm{NaBH}_{4}$ and $\mathrm{HCl}$ concentrations $(0.05 \%-2.0 \%$, respectively) and from different reaction media, i.e., citric acid at $\mathrm{pH} 4.5$ and acetic acid for lonely As(III) determination, and (2) total i-As determination in the TGA medium or after pre-reduction with a KI-ascorbic acid mixture. Except for the acetic acid based procedure, all of them could be used to distinguish between these two forms. Acetic acid was not selective enough for the determination of 
As(III) coexisting with As(V). The best analytical performance was achieved using the procedure with the citric buffer and KI-ascorbic pre-reduction for As(III) and total i-As, respectively. The obtained LODs were two times better than those achieved with the two remaining procedures. On the other hand, in contrast to the pre-reduction of As(V) with KI-ascorbic, the determination of As(III) and As(V) in the presence of TGA medium, with virtually the same responses of both species, could be achieved just after adding this acid to sample solutions. With the KI-ascorbic acid mixture, the conversion of As(V) into As(III) was completed within $1 \mathrm{~h}$. Citric acid was also found to be the most effective reagent in the determination of As(III) alone in soil extracts by HG-AFS [32]. Among the other tested media, including low concentration acetic and tartaric acids, $\mathrm{HNO}_{3}$ and $\mathrm{HCl}$, only when citric acid was used, the unwanted presence of As(V) could be virtually eliminated. Similarly, Lehmann et al. [70] succeeded in speciating inorganic As in plankton samples using a metallic furnace atomizer with HG-AAS by controlling the reaction medium and avoiding the reduction of As(V) to As(III). Importantly, measurements of As(III) and total i-As (as As(III) after pre-reduction) were carried out using the same mild conditions for HG, such as a slightly acidic media (a citrate buffer at pH 4.5 was used) and a low $\mathrm{NaBH}_{4}$ concentration (0.1\%). Among the various pre-reducing agents tested, including KI-ascorbic acid, $\mathrm{Na}_{2} \mathrm{~S}_{2} \mathrm{O}_{3}$, L-cysteine and thiourea, it was found that L-cysteine was able to reduce As(V) to As(III) at $\mathrm{pH}$ 4.5. This was probably due to the strong affinity of the As(III) to -SH group present in the L-cysteine structure, which may change the mechanism of reduction and promote the conversion of As(V) to As(III).

Interestingly, in two works [57,59], $\mathrm{AsH}_{3}$ was generated selectively for As(III) in the presence of 8-hydroxyquinoline under $\mathrm{HCl}$ acidity. Possibly, an ion associate of $\mathrm{As}(\mathrm{V})$ with 8-hydroxyquinoline was likely formed, making As(V) unreactive. On the other hand, 8-hydroxyquinoline had an enhancing effect on the responses of both $\mathrm{As}(\mathrm{III})$ and $\mathrm{As}(\mathrm{V})$ pre-treaded with KI/KI-ascorbic acid before HG. Additionally, the presence of 8-hydroxyquinoline in the reaction medium lessened the interference from transition metals occurring in the generation of arsine. The proposed strategy was found to be attractive in analyses of herbaceous plants by HG-AFS [57] and dietary supplements by HG-AAS [59].

Regarding the different behavior of $\mathrm{As}(\mathrm{III})$ and $\mathrm{As}(\mathrm{V})$ in the HG process, speciation was also made using proportional equations corresponding to two different measurement conditions for the same sample (acid- $\mathrm{NaBH}_{4}$ combination) $[17,29,49,50,56]$. In three cited works $[49,50,56]$, these two different conditions referred to direct measurements of diluted sample extracts and measurements after the previous pre-reduction step with KI-ascorbic acid-hydroxylamine hydrochloride [49], KI-ascorbic acid [50] or KI-thiourea [56] mixtures. This was successfully applied to determine both i-As species in various materials, including milk [49], mushrooms [50] and TCM herbs [56] by HG-AFS/AAS. To establish these simultaneous equations, two HG reaction conditions combined with L-cysteine for pre-reduction were also used by Wang and Tyson [17] to determine As(III) and As(V) quantities in fresh water samples (sea, tap, pound) by HG-AFS. To overcome interference from Fe(III) in the case of seawater, standard addition calibration curves were employed for As quantification. Finally, Cai et al. [29] obtained a proportional dependence of As species using citric acid for As(III) and thiourea-ascorbic acid for total i-As. The methodology was used to distinguish As(III) and As(V) in tea by HG-AFS.

This subtraction-based speciation scheme could also be simplified and realized without the need of a previous pre-reduction step for total i-As. However, this was possible only for the case when As(III) and $\mathrm{As}(\mathrm{V})$ species responded similarly under applied HG conditions. Anthemidis et al. [20] reported that $\mathrm{AsH}_{3}$ could be selectively generated either for $\mathrm{As}$ (III) or total i-As using different $\mathrm{HCl}$ and $\mathrm{NaBH}_{4}$ concentrations. As a result, the authors proposed two different pairs of $\mathrm{HCl}$ and $\mathrm{NaBH}_{4}$ concentrations, i.e., $1.5 \mathrm{~mol} \mathrm{~L}^{-1} \mathrm{HCl}-0.5 \% \mathrm{NaBH}_{4}$ and $9.0 \mathrm{~mol} \mathrm{~L}^{-1} \mathrm{HCl}-3.0 \% \mathrm{NaBH}_{4}$ for the selective determination of As(III) and total i-As, respectively, in natural water samples (river, lake, tap) by HG-AAS. Similarly, as shown in [35,72], As(III) and As(V) can be determined with the same sensitivity at $0.3 \%$ [72] or $1.0 \%$ [35] $\mathrm{NaBH}_{4}$ in the medium of $0.48 \%$ TGA [72] or $6 \mathrm{~mol} \mathrm{~L}{ }^{-1} \mathrm{HCl}$ [35] in environmental [72] or glass [35] samples by HG-AAS; no additional pre-reduction step was required. 
The main drawback of the proposed speciation schemes could be that the presence of o-As species in the sample matrix, especially MMA and DMA, which generate their respective hydrides under chosen conditions, could selectivity affect the measurements of As(III) and As(V). This is evident when a -SH group containing pre-reductants such as L-cysteine or TGA is used for As(V) pre-reduction. Both result in the same response for all four As species, leading to overestimations of $\mathrm{As}(\mathrm{V})$ concentrations [2].

In some works, the contribution of methylated As species (o-As) to the quantified i-As concentration was assessed by evaluating the interference of DMA and MMA in the As signal under selected pre-reduction and HG reaction conditions [2,12,17,27,38,47,59,83]. It was found that the increase in $\mathrm{HCl}$ concentration $\left(9 \mathrm{~mol} \mathrm{~L}{ }^{-1}\right)$ and the application of $\mathrm{KI}$ at the pre-reduction step successfully diminished the negative effects coming from the presence of both DMA and MMA during inorganic As speciation in wine by HG-AAS [27]. In another work [59], the selectivity of As(III) and As(V) measurements in dietary supplements by HG-AAS, i.e., with no interference from either methylated As species, was guaranteed under $1-5 \% \mathrm{HCl}$ acidity conditions in the presence of 8-hydroxyquinoline and KI, added to pre-reduce As(V) into As(III). In two other works [38,47], using $0.1 \% \mathrm{NaBH}_{4}$ and $10 \mathrm{~mol} \mathrm{~L}^{-1} \mathrm{HCl}$ in combination with $\mathrm{KI}$-ascorbic acid for pre-reduction, it was possible to separately determine As(III) and i-As in rice and rice products by HG-AAS. However, the absence of MMA in the sample was considered; DMA remained undetected, as evidenced by its signal being close to that of the blank. Interference effects coming from DMA and MMA were also noted; therefore, methods developed for the speciation of i-As were proposed and used for samples with negligible or no methylated As compounds [2,12,17,83]. Optionally, to overcome the interference effects from DMA and MMA, solid phase extraction (SPE) with specific sorbents were used to separate i-As from o-As before HG $[23,41-43,55,79]$ (see point 5.1). Organic As could also be removed after the HG reaction by freezing out in a liquid nitrogen trap installed between a gas/liquid separator and a detection device [83]. Occasionally, the amount of o-As was defined by subtracting the total i-As content from the total As $\left(\mathrm{As}_{\mathrm{T}}\right)$ content determined after complete sample decomposition by wet digestion $[27,29,45,50,56,57]$.

\subsubsection{Operational Speciation, i.e., Dealing Only with i-As Determination (total As(III) and As(V))}

This approach compromises the inorganic As species present in the sample, and is recommended when inter-conversion between $\mathrm{As}(\mathrm{III})$ and $\mathrm{As}(\mathrm{V})$ is not an issue. The toxicity of As depends on the presence of both inorganic forms; hence, conditions for the selective determination of i-As, i.e., total As(III) and As(V), in the presence of other organoarsenic compounds, including methylated forms and As-sugars, are of special interest.

Depending on the reagents applied at the sample preparation step, e.g., a low concentration of $\mathrm{HNO}_{3}$ (alone [46] or combined with $\mathrm{H}_{2} \mathrm{O}_{2}[44,53,54]$ ) or aqua regia [45]), As can be present in sample extracts in the form of $\mathrm{As}(\mathrm{III})$ and $\mathrm{As}(\mathrm{V})$ or as i-As, being exclusively $\mathrm{As}(\mathrm{V})$ after the conversion of all $\mathrm{As}(\mathrm{III})$ to $\mathrm{As}(\mathrm{V})$ during extraction. For the selective determination of i-As by HG, i-As species should be present as a single species, i.e., as $\mathrm{As}(\mathrm{III})$ or $\mathrm{As}(\mathrm{V})$, rather than a mixture of $\mathrm{As}(\mathrm{III})$ and $\mathrm{As}(\mathrm{V})$. Since As(III) reacts more effectively with $\mathrm{NaBH}_{4}$ than $\mathrm{As}(\mathrm{V})$, the pre-reduction of $\mathrm{As}(\mathrm{V})$ to $\mathrm{As}(\mathrm{III})$ prior to HG is preferred. As a result, total i-As is measured in the form of As(III). Selected optimal parameters developed for the selective determination of traces of i-As in rice by HG-ICP-MS [44], HG-ICP-OES [45] or HG-AAS [46], and in more complex matrices, i.e., samples of marine origin such as seaweed, by HG-ICP-MS [44,53,54], are summarized in Table 3. 
Table 3. Non-chromatographic procedures for operational speciation, i.e., i-As determination (total As(III) and As(V)).

\begin{tabular}{|c|c|c|c|c|c|c|}
\hline Matrix & Species & Sample Preparation ${ }^{a}$ & Speciation Procedures & Detection & $\mathrm{LOD}^{\mathrm{b}}, \mu \mathrm{g} \mathrm{L}^{-1}$ & Ref. \\
\hline rice, seaweed & i-As $($ as $\mathrm{As}(\mathrm{V}))$ & $\begin{array}{c}\text { MAE } \\
\left(1-2 \% \mathrm{HNO}_{3}-3 \% \mathrm{H}_{2} \mathrm{O}_{2}\right)\end{array}$ & S: $3 \% \mathrm{H}_{2} \mathrm{O}_{2}, \mathrm{~A}: 5 \mathrm{M} \mathrm{HCl}, \mathrm{R}: 2 \% \mathrm{NaBH}_{4}$ & HG-ICP-MS & 0.006 & [44] \\
\hline rice & i-As (as As(III)) & $\begin{array}{l}\text { US } \\
\text { (aqua regia) }\end{array}$ & $\begin{array}{c}\text { S: } 1.25 \mathrm{M} \text { aqua regia-KI-ascorbic acid-3 M } \\
\mathrm{HCl}, \mathrm{A}: 10 \mathrm{M} \mathrm{HCl}, \mathrm{R}: 1 \% \mathrm{NaBH}_{4}\end{array}$ & HG-ICP-OES & $0.28 \mathrm{ng} \mathrm{g}^{-1}$ & [45] \\
\hline rice & i-As (as As(III)) & $\begin{array}{c}\text { MAE } \\
\left(0.28 \mathrm{M} \mathrm{HNO}_{3}\right)\end{array}$ & $\begin{array}{c}\text { S: } 0.28 \mathrm{M} \mathrm{HNO}_{3}-\mathrm{KI} \text {-ascorbic acid-3 M HCl, } \\
\text { A: } 1.2 \mathrm{M} \mathrm{HCl}^{\mathrm{R}} \mathrm{R}: 0.1 \% \mathrm{NaBH}_{4}\end{array}$ & HG-AAS & $16 \mathrm{ng} \mathrm{g}^{-1 \mathrm{c}}$ & [46] \\
\hline seafood, seaweed & i-As (as As(V)) & $\begin{array}{c}\text { MAE } \\
\left(2 \% \mathrm{HNO}_{3}-3 \% \mathrm{H}_{2} \mathrm{O}_{2}\right)\end{array}$ & S: $3 \% \mathrm{H}_{2} \mathrm{O}_{2}, \mathrm{~A}: 8 \mathrm{M} \mathrm{HCl}, \mathrm{R}: 2 \% \mathrm{NaBH}_{4}$ & HG-ICP-MS & 0.01 & {$[53]$} \\
\hline seaweed & i-As (as As(V)) & $\begin{array}{c}\text { MAE } \\
\left(2 \% \mathrm{HNO}_{3}-3 \% \mathrm{H}_{2} \mathrm{O}_{2}\right)\end{array}$ & S: $3 \% \mathrm{H}_{2} \mathrm{O}_{2}, \mathrm{~A}: 5 \mathrm{M} \mathrm{HCl}, \mathrm{R}: 2 \% \mathrm{NaBH}_{4}$ & HG-ICP-MS & 0.06 & [54] \\
\hline
\end{tabular}

HG-AAS: hydride generation atomic absorption spectrometry. HG-ICP-OES: hydride generation inductively-coupled plasma optical emission spectrometry. HG-ICP-MS: hydride generation inductively-coupled plasma mass spectrometry. i-As: the inorganic tri- and pentavalent As species (As(III) and As(V)). M: mol L ${ }^{-1}$. MAE: microwave-assisted extraction. S: sample solution. A: additional acid (carrier) solution. R: reductant solution. US: ultrasonication in an ultrasound water bath. a Detailed information about sample preparation procedures described in point $4 .{ }^{\mathrm{b}}$ For $\mathrm{As}(\mathrm{V})$ as As(III) without or after previous pre-reduction step. ${ }^{\mathrm{c}}$ In the original sample taking into account the amount of sample and the final dilution employed in the proposed procedure. 
Generally, the selectivity of i-As determination is based on the application of a high concentration of $\mathrm{HCl}$ (5-10 $\left.\mathrm{mol} \mathrm{L}^{-1}\right)$ for $\mathrm{HG}$ and a KI-ascorbic acid mixture for pre-reduction of $\mathrm{As}(\mathrm{V})$. By careful selection of flow rates of solutions, a lower optimum $\mathrm{HCl}$ concentration $\left(1.2 \mathrm{~mol} \mathrm{~L}^{-1}\right)$ can be achieved [46]. Unfortunately, in a variant of strong sample acidity required for selective HG, L-cysteine could not be used at the pre-reduction step [54]. The concentration of $\mathrm{NaBH}_{4}$ used for the determination of i-As varied (0.1-2.0\%); nevertheless, its higher concentrations had to be used when $\mathrm{HNO}_{3}$ combined with $\mathrm{H}_{2} \mathrm{O}_{2}$ was used to extract both As species. At least $2 \% \mathrm{NaBH}_{4}$ was needed to overcome the interference effects which occurred in $\mathrm{HG}$ for $\mathrm{As}(\mathrm{V})$ coming from the presence of $\mathrm{H}_{2} \mathrm{O}_{2}$ in sample extracts left after extraction. Additionally, $\mathrm{H}_{2} \mathrm{O}_{2}$ interfered with pre-reduction of $\mathrm{As}(\mathrm{V})$ by $\mathrm{KI}$, which made it impossible to determine the i-As content in real samples in the form of As(III). Accordingly, in these works $[44,53,54]$, total $\mathrm{i}$-As was measured as $\mathrm{As}(\mathrm{V})$ after oxidation with $\mathrm{H}_{2} \mathrm{O}_{2}$. In contrast, such an effect was not observed when aqua regia- [45] or $\mathrm{HNO}_{3}$-based [46] sample extraction procedures were used to release As species from sample matrices.

The main goal of these optimization studies was to provide a contribution of all hydride-active methylated As species to the i-As signal as low as possible. Besides adequate reducing conditions, sample preparation in terms of the reagents used for extraction was helpful in improving the selectivity of i-As determination in the presence of coexisting o-As species. In three works $[44,53,54]$, high concentrations of $\mathrm{HCl}$ for $\mathrm{HG}$ and $\mathrm{H}_{2} \mathrm{O}_{2}$ in samples of the same concentration, as used for extraction ( $3 \%)$, led to the selective conversion of $\mathrm{i}$-As to volatile arsine, while HG from DMA was substantially inhibited (less than 1-3\% of the i-As signal) (see Table 3). Unfortunately, these schemes introduced some errors due to a significant contribution of MMA to the i-As signal (21-43\%). However, it is argued that MMA is normally absent or present in trace amounts in most samples of rice and seafood, and hence, would not affect the quantification of i-As in these materials. Interference from a rich As-sugars matrix in the determination of i-As with HG was also negligible; therefore, the described methodologies were suitable as quick reliable screening methods for i-As determination in seaweed [54]. Similar results were achieved for the i-As species measured in sample extracts containing $0.28 \mathrm{~mol} \mathrm{~L}^{-1}$ $\mathrm{HNO}_{3}$, used to extract As species from various types of rice (paddy, brown, polished, parboiled) [46]. In one work [45], where aqua regia was used for extraction, interferences coming from both DMA and MMA were successfully eliminated, making it possible to reliably differentiate between i-As and o-As, and to selectively determine traces of $\mathrm{i}$-As in brown rice by HG-ICP-OES. The presence of $1.25 \mathrm{~mol} \mathrm{~L}^{-1}$ aqua regia in the sample extracts and a high concentration of $\mathrm{HCl}$ for the $\mathrm{HG}$ reaction were advantageous to limit the activity of o-As during HG, but not i-As.

\subsection{Case of Inorganic As(III) and As(V) and Organic Arsenic (DMA and MMA)—Speciation and Fractionation Protocols}

As presented above, the response of As achievable in the HG reaction strongly depends not only on the oxidation state (III/V) and experimental conditions, but also on the nature of hydride-active As species (inorganic/organic). Accordingly, distinguishing between tri- and penta- valent arsenicals, provided by selective HG, can expand speciation analyses of $\mathrm{i}$-As to its methylated forms (DMA, MMA). Undoubtedly, non-chromatographic approaches to the differentiation of four As species by means of HG are the most desirable, but also the most challenging. Their development has to be proceeded by the careful optimization of experimental parameters, being appropriate for each As species present in the sample solution.

Based on the different reactivities of all four As species under special pre-reducing and HG reaction conditions, procedures for species-selective HG of As can be evaluated using the same rules as those provided for i-As analysis, i.e., $\mathrm{pH}$ specific $\mathrm{HG}$ reaction or selective conditions (a proper acid- $\mathrm{NaBH}_{4}$ combination and a pre-reductant). By combining the responses obtained for these procedures (simple mathematical subtraction or a series of independent proportional equations), protocols for the non-chromatographic speciation of As and determination of its species at a trace level in various matrices, including food $[39,40,52,81]$, beverages $[3,7,28]$ and environmental $[3,13,75-77]$ 
samples, have been proposed. In addition to individual speciation $[3,7,28,39,51,52]$, procedures to fractionate As were also evaluated (operational speciation) by distinguishing between species of the same nature, i.e., i-As versus o-As [71] or $\mathrm{As}_{\text {toxic }}$ versus $\mathrm{As}_{\text {non-toxic }}$ [52,71]. The fraction of $\mathrm{As}_{\text {toxic }}$ indicates hydride-active species $\left(\mathrm{As}_{\mathrm{h}}\right)$, i.e., the sum of $\mathrm{As}(\mathrm{III})+\mathrm{As}(\mathrm{V})+\mathrm{DMA}+\mathrm{MMA}$, while the content of $A s_{\text {non-toxic }}$ refers to unreactive As forms toward HG $\left(\mathrm{As}_{\mathrm{nh}}\right)$. Typically, the latter is determined after sample digestion by the difference between the total As content $\left(\mathrm{As}_{\mathrm{T}}\right)$ and $\mathrm{As} \mathrm{s}_{\mathrm{h}}$. Variants among sensitive determinations of three- and four-species of As are detailed in Table 4. 
Table 4. Non-chromatographic speciation and fractionation protocols for the determination of various As species by HG.

\begin{tabular}{|c|c|c|c|c|c|c|}
\hline Matrix & Species & Sample Preparation ${ }^{a}$ & Speciation Procedures & Detection & $\mathrm{LOD}^{b}, \mu \mathrm{g} \mathrm{L}^{-1}$ & Ref. \\
\hline $\begin{array}{l}\text { cereals, fish, } \\
\text { vegetables }\end{array}$ & $\begin{array}{c}\mathrm{As}(\mathrm{III}) \\
\mathrm{As}(\mathrm{V}) \\
\mathrm{DMA} \\
\mathrm{MMA} \\
\mathrm{t}-\mathrm{As} \mathrm{s}_{\text {toxic }} \\
\mathrm{t}-\mathrm{As} \text { non-toxic }\end{array}$ & $\begin{array}{c}\text { US }\left(3 \mathrm{M} \mathrm{HNO}_{3} \text { or } 1 \mathrm{M}\right. \\
\mathrm{H}_{3} \mathrm{PO}_{4}-0.1 \% \text { Triton } \\
\mathrm{X}-114+0.1 \% \text { EDTA })\end{array}$ & 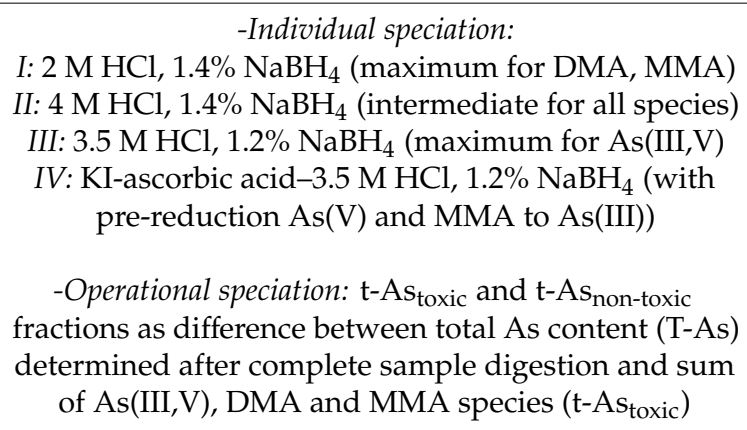 & HG-AFS & $\begin{array}{l}\text { As(III): } 0.62-3.1 \mathrm{ng} \mathrm{g}^{-1 \mathrm{c}} \\
\text { As(V): } 0.9-3.0 \mathrm{ng} \mathrm{g}^{-1 \mathrm{c}} \\
\text { DMA: } 1.5-1.8 \mathrm{ng} \mathrm{g}^{-1 \mathrm{c}} \\
\text { MMA: } 0.6-5.4 \mathrm{ng} \mathrm{g}^{-1 \mathrm{c}}\end{array}$ & {$[39,51,52]$} \\
\hline rice & $\begin{array}{l}\text { As(III) } \\
\text { As(V) } \\
\text { DMA } \\
\text { MMA }\end{array}$ & US $\left(1 \% \mathrm{HNO}_{3}\right)$ & $\begin{array}{c}\text { I: } 0.06 \mathrm{M} \text { citric acid (maximum for As(III)+DMA) } \\
\text { II: } \mathrm{K}_{2} \mathrm{~S}_{2} \mathrm{O}_{8}-0.06 \mathrm{M} \text { citric acid (maximum for DMA) } \\
\text { III: } \mathrm{L} \text {-cysteine-ascorbic acid-0.06 M citric acid } \\
\text { (maximum for As(III)+As(V)+DMA) } \\
\text { IV: thiourea-ascorbic acid-5\% } \mathrm{HCl} \text { (pre-reduction of } \\
\text { As(V), DMA and MMA to As(III)) } \\
\text { A: } 1.6 \mathrm{M} \mathrm{HCl-citrate} \mathrm{buffer} \mathrm{(pH} \mathrm{4.8),} \mathrm{R:} 2 \% \mathrm{KBH}_{4} \\
\text { I. As(III): }\end{array}$ & HG-AFS & $\begin{array}{l}\text { As(III): } 0.21 \mu \mathrm{g} \mathrm{kg}^{-1} \\
\text { As(V): } 0.52 \mu \mathrm{g} \mathrm{kg}^{-1} \\
\text { DMA: } 0.65 \mu \mathrm{g} \mathrm{kg}^{-1} \\
\text { MMA: } 0.9 \mu \mathrm{g} \mathrm{kg}^{-1}\end{array}$ & [52] \\
\hline ground water & $\begin{array}{l}\text { As(III) } \\
\text { As(V) } \\
\text { DMA }\end{array}$ & direct analysis & $\begin{array}{c}\text { S: oxalate buffer (pH 4-4.5), R: } 0.6 \% \mathrm{NaBH}_{4} \\
\text { II. } A s(I I I)+A s(V): \\
\text { S: KI, A: } 6 \mathrm{M} \mathrm{HCl}, \mathrm{R}: 0.6 \% \mathrm{NaBH}_{4} \\
\text { III. DMA: }\end{array}$ & HG-AAS & $\begin{array}{l}\text { As(III): } 0.1 \\
\text { As(III,V): } 0.1 \\
\text { DMA: } 0.19\end{array}$ & [7] \\
\hline natural waters & $\begin{array}{l}\text { As(III) } \\
\text { As(V) } \\
\text { DMA } \\
\text { MMA }\end{array}$ & direct analysis & $\begin{array}{c}\text { S: L-cysteine, A: } 1.5 \mathrm{M} \mathrm{HCl} \text { R: } 0.6 \% \mathrm{NaBH}_{4} \\
\text { I. As(III)+As(V)+MMA: } \\
\text { S: KI-0.1 M HCl, A: } 1 \mathrm{M} \mathrm{HCl}, \mathrm{R}: 0.6 \% \mathrm{NaBH}_{4} \\
\text { II. } A s(I I I)+D M A: \\
\text { S: } 0.1 \mathrm{M} \mathrm{HCl} \text { A: } 6 \mathrm{M} \mathrm{CH} \mathrm{H}_{3} \mathrm{COOH}, \mathrm{R}: 0.6 \% \mathrm{NaBH}_{4} \\
\text { III. } A s(I I I)+A s(V)+D M A: \\
\text { S: } \mathrm{KI}-0.1 \mathrm{M} \mathrm{HCl} \text { A: } 6 \mathrm{M} \mathrm{CH} \text { COOH, R: } 0.6 \% \mathrm{NaBH}_{4} \\
\text { IV. } A s(I I I)+A s(V)+D M A+M M A: \\
\text { S: KI-0.1 M HCl, A: } 1 \mathrm{M} \text { tartaric acid, R: } 0.6 \% \mathrm{NaBH}_{4}\end{array}$ & HG-AAS & 0.1 & [3] \\
\hline
\end{tabular}


Table 4. Cont.

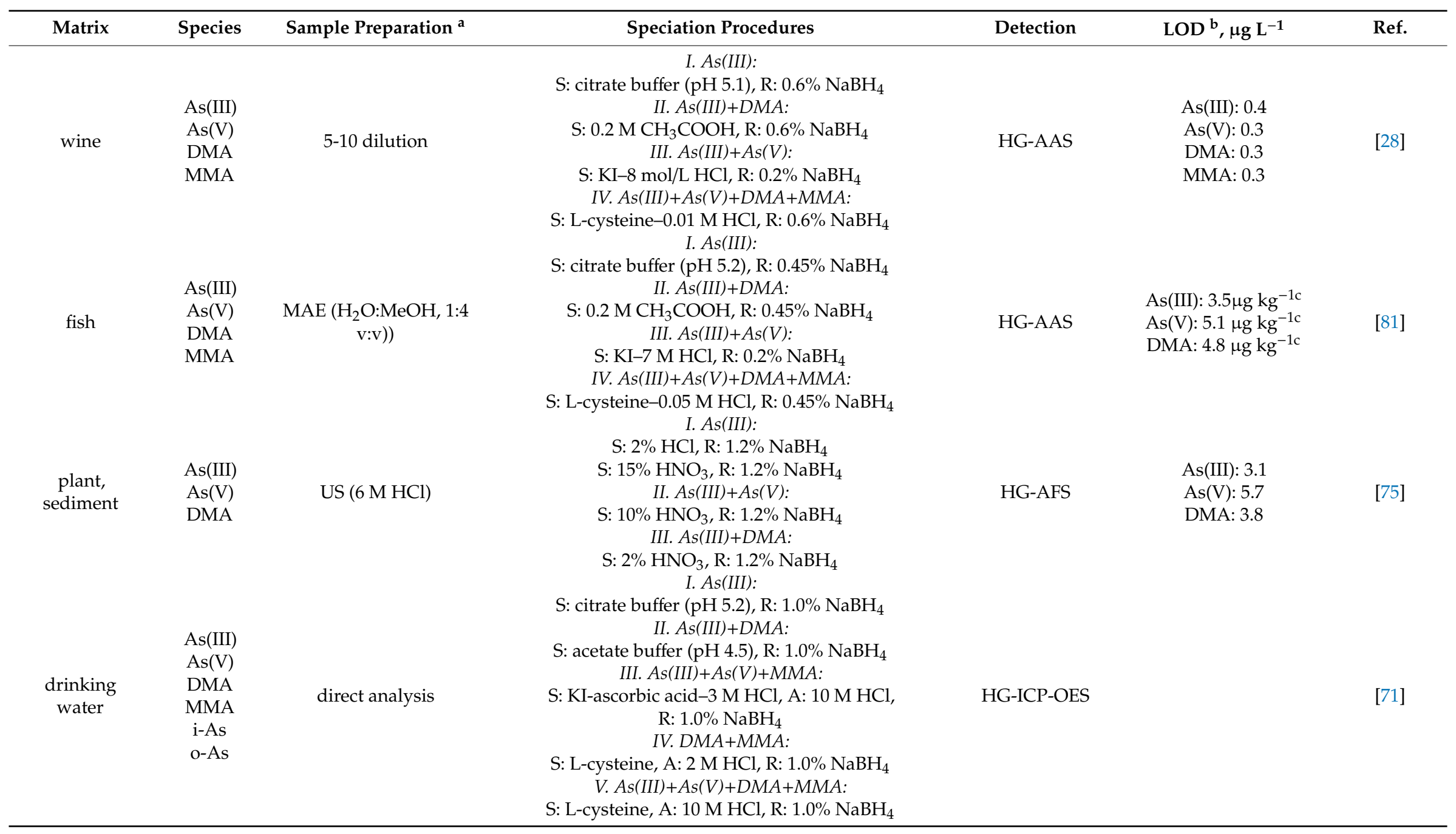


Table 4. Cont.

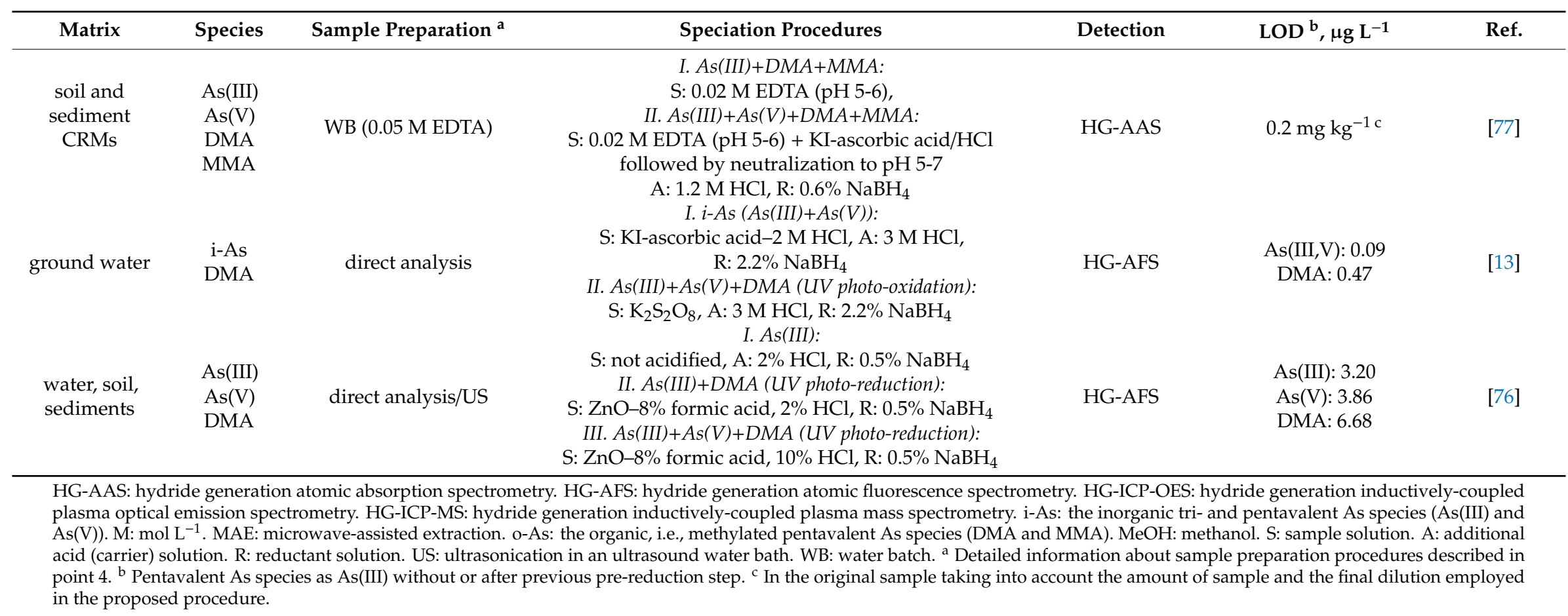


As shown in Table 4, to selectively generate hydrides for each As species in the reaction with $\mathrm{NaBH}_{4}$, the reaction medium-i.e., the type of acid and its acidity, including inorganic $(\mathrm{HCl}[3,7,13,28$, $39,51,52,71,75-77], \mathrm{HNO}_{3}$ [75]) and carboxylic acids (e.g., tartaric [3,77], acetic [28,71,81], citric [40,71], formic [76], oxalic [77], malic [77]) or buffers (citrate at pH 5 [28,40,71,81]), oxalate at pH 4.5 [7], acetate at $\mathrm{pH} 4.5$ [71]), the concentration of $\mathrm{NaBH}_{4}[13,28,39,51,52,71]$, and the kind of the pre-reducing agent employed at the step of the sample pre-treatment (mostly KI/KI-ascorbic acid $[3,7,28,39,51,52,71,77,81]$, thiourea-ascorbic acid [40,71] and L-cysteine [7,28,40,71,81]—was typically controlled.

Unfortunately, in contrast to the speciation of inorganic As only, one uniform strategy when all four forms are speciated is difficult to establish. However, some selective reaction media for speciation purposes have been recommended. As shown in [28,71,81], which focus on As speciation in wine and fish by HG-AAS [28,81] or drinking water by HG-ICP-OES [71], the determination of only As(III) could be achieved in the presence of a citrate buffer $(\mathrm{pH} 5)$, while the presence of a low concentration of acetic acid [28,81] or an acetate buffer ( $\mathrm{pH} 4.5)$ [71] ensured the generation of arsines for As(III) and DMA. The sum of i-As (As(III) and As(V)) alone [28,81] or together with MMA (i-As+MMA) [71] could be determined after the pre-reduction of V-state As to As(III) with KI/KI-ascorbic and 7-10 mol L ${ }^{-1} \mathrm{HCl}^{-}$ for the HG reaction. Interestingly, the sum of o-As species, i.e., (DMA+MMA), could be selectively determined after their pre-reduction with L-cysteine, followed by $\mathrm{HG}$ in $2 \mathrm{~mol} \mathrm{~L}^{-1} \mathrm{HCl}$ [71]. Finally, L-cysteine, used for the pre-reduction of As(V), DMA and MMA to As(III), enabled us to determine total As. Nevertheless, quantitative pre-reduction was reached under completely different HG reaction conditions in terms of the $\mathrm{HCl}$ concentration, i.e., $0.01-0.05 \mathrm{~mol} \mathrm{~L}^{-1}$ [28,81] and $10 \mathrm{~mol} \mathrm{~L}^{-1}$ [71]. Otherwise, all four As forms could be speciated by HG-AAS in natural waters (sea, underground, drinking) by conducting the reduction reaction at a fixed $\mathrm{NaBH}_{4}$ concentration $(0.6 \%)$, using different reaction media ( $\mathrm{HCl}$, acetic and tartaric acids) and the pre-reduction step with $\mathrm{KI}[3]$.

In contrast to a popular speciation method by subtraction, it seems that the strategy with HG reaction conditions under which hydrides of As species are generated with different efficiencies may be much easier to establish, as evidenced in several works cited here. In three of them, the determination of all four As forms (As(III,V), DMA and MMA) in fish [52], cereals (rice, wheat semolina) [39] and vegetables (chard, aubergine) [51] by HG-AFS was carried out using a series of independent proportional equations corresponding to four different reduction conditions (I-IV) based on various $\mathrm{HCl}$ and $\mathrm{NaBH}_{4}$ concentrations, i.e., $2-4 \mathrm{~mol} \mathrm{~L}^{-1} \mathrm{HCl}$ and 1.2-1.4\% $\mathrm{NaBH}_{4}$. An additional pre-treatment with KI-ascorbic acid was applied in one case (condition IV) to reduce As(V) and MMA to As(III). Importantly, non-reducible (non-hydride reactive) As species during HG, such as non-toxic AsB, remained unchanged [51,52]. In the same way, i.e., with the aforementioned linear equation approach, all four As forms were speciated and determined in rice by HG-AFS [40]. It was possible to find four different sample pre-treatment procedures to selectively generate As hydrides in the same HG reaction conditions $\left(1.6 \mathrm{~mol} \mathrm{~L}^{-1} \mathrm{HCl}\right.$-citrate buffer $\left.(\mathrm{pH} 4.8)-\mathrm{KBH}_{4}\right)$. Several common pre-reducing (KI, L-cysteine, thiourea, ascorbic acid) and preoxidizing $\left(\mathrm{H}_{2} \mathrm{O}_{2}, \mathrm{KMnO}_{4}, \mathrm{~K}_{2} \mathrm{~S}_{2} \mathrm{O}_{8}\right)$ agents that would make it possible to pre-reduce As(V), DMA and MMA to As(III) or oxidize As(III) to As(V) in the sample solution acidified to $0.06 \mathrm{~mol} \mathrm{~L}^{-1}$ citric acid were tested. As a result, in the presence of a low concentration of citric acid alone, a maximum signal for As(III)+DMA was provided (condition I); $\mathrm{K}_{2} \mathrm{~S}_{2} \mathrm{O}_{8}$ completely oxidized As(III), but it did not degrade DMA, and hence, allowed for selective HG for DMA, importantly, without any interference effect from As(V) and MMA (condition II); the use of L-cysteine-ascorbic acid made it possible to determine the sum of As(III), As(V) and DMA, without any contribution of MMA to the overall signal (condition III); finally, the determination of all As species by $\mathrm{HG}$ was most effective in the presence of $\mathrm{HCl}$ and thiourea-ascorbic acid for pre-reduction (condition IV). It is noteworthy that the results for L-cysteine were similar to those presented in work [70], and proved the pre-reducing potential of this reagent in a citric acid medium ( $\mathrm{pH} 4.5-4.8)$.

An interesting approach to the determination of the sum of As(III)+DMA+MMA and the sum of all toxicologically relevant hydride-active As species (As(III)+As(V)+DMA+MMA) in EDTA extracts of soil and sediment samples by HG-AAS was evaluated in [77]. An additional pre-treatment with 
$\mathrm{KI}$-ascorbic acid was applied to reduce $\mathrm{As}(\mathrm{V})$ to $\mathrm{As}(\mathrm{III})$, and then the $\mathrm{As}(\mathrm{V})$ concentration was calculated by the appropriate difference. Moreover, the influence of various types of carboxylic acids, their aminoand hydroxo-derivatives and monosaccharides on the efficiency of the HG process was investigated. Observations showed that EDTA, ascorbic acid, glucose and fructose leveled and equalized the responses of As(III), DMA and MMA at $\mathrm{pH} 5-7$, and furthermore, that ascorbic acid, glucose and fructose maintained their leveling effect at $\mathrm{pH} 1.3-2\left(0.01-0.05 \mathrm{~mol} \mathrm{~L}^{-1} \mathrm{HCl}\right)$.

In two other works $[13,76]$, sample pre-treatment with UV irradiation resulted in photo-oxidation or photo-reduction processes that promoted the inter-conversion of As species prior to HG. Chaparro et al. [13] proposed an approach for the selective determination of total i-As and DMA in ground water samples using an automated HG-AFS system. In the first procedure, total As (the sum of As(III), $\mathrm{As}(\mathrm{V})$ and DMA) was determined after UV irradiation of the sample in a $\mathrm{K}_{2} \mathrm{~S}_{2} \mathrm{O}_{8}$ medium and after photo-oxidation of all As species to inorganic $\mathrm{As}(\mathrm{V})$. In the second one, total i-As was measured directly (i.e., the photo-oxidation step was omitted) after the previous pre-reduction of $\mathrm{As}(\mathrm{V})$ to As(III) with KI-ascorbic acid. The DMA concentration was calculated by the difference. Pinheiro et al. [76] reported that all three As forms were speciated and determined by HG-AAS by coupling a photo-reduction system with a HG manifold. The effective photo-reduction of DMA and As(V) to inorganic As(III) was achieved by using UV treatment (catalyzed by ZnO nanoparticles) in a formic acid medium. Various reaction conditions, in terms of the $\mathrm{HCl}$ concentration, were used for the selective generation of individual hydrides. With $2 \% \mathrm{HCl}$ for the $\mathrm{HG}$ reaction, $\mathrm{As}(\mathrm{III})$ was directly determined, while As(III)+DMA could be determined after the photo-reduction step. By applying $10 \% \mathrm{HCl}$ and the same photo-reduction process, $\mathrm{As}(\mathrm{III})+\mathrm{As}(\mathrm{V})+\mathrm{DMA}$ were quantified. The proposed strategies were suitable for As speciation in environmental samples such as water and soil or sediments (after US-assisted extraction).

However, Pinheiro et al. [75] and Akter et al. [7] demonstrated that As(III), As(V) and DMA could be also selectively determined without UV irradiation of samples. Accordingly, the selective generation of $\mathrm{As}(\mathrm{III}), \mathrm{As}(\mathrm{V})$ and DMA hydrides was possible using $1.2 \% \mathrm{NaBH}_{4}$ and changing the acid type and its concentration [75]. It is noteworthy that no additional pre-reducing step was required. For each species, different experimental reduction conditions, based on different $\mathrm{HCl}$ and $\mathrm{HNO}_{3}$ concentrations, were used. Four speciation procedures were developed and their reliability was verified. Employing various $\mathrm{HCl}$ concentrations, i.e., $2 \%$ and $10 \%$, $\mathrm{As}(\mathrm{III})$ and i-As $(\mathrm{As}(\mathrm{III})+\mathrm{As}(\mathrm{V}))$ were determined, respectively. The content of $\mathrm{As}(\mathrm{V})$ was obtained by calculating the difference. Employing various $\mathrm{HNO}_{3}$ concentrations, i.e., $15 \%$ and $2 \%, \mathrm{As}(\mathrm{III})$ and $\mathrm{As}(\mathrm{III})+\mathrm{DMA}$, were determined, respectively. Similarly to $\mathrm{As}(\mathrm{V})$, by the difference in responses found for 2 and $15 \% \mathrm{HNO}_{3}$, the exact content of DMA was calculated. The proposed speciation approach was applicable for As speciation in sediment and plant samples by HG-AFS. Akter et al. [7] used a well-known procedure for i-As speciation based on selective As(III) determination at $\mathrm{pH} 4.5$ (oxalate buffer) and total i-As in the $\mathrm{HCl}$ medium $\left(6 \mathrm{~mol} \mathrm{~L}^{-1}\right)$ after pre-reduction of $\mathrm{As}(\mathrm{V})$ to $\mathrm{As}(\mathrm{III})$ with $\mathrm{KI}$. Otherwise, the reaction medium of $1.5 \mathrm{~mol} \mathrm{~L}^{-1} \mathrm{HCl}$ and the sample pre-treatment with L-cysteine allowed made it possible to determine the DMA alone. The same $\mathrm{NaBH}_{4}$ concentration $(0.6 \%)$ was used in all three procedures. The developed methodology was applied for As speciation in ground water samples by HG-AAS.

\section{Pre-Concentration and/or Separation}

The concentrations of As in environmental, food and biological samples are usually very low (see Table 1). The HG technique, coupled with atomic spectrometric detectors, is a sensitive analytical tool for the determination of traces of As. However, when handling ultratrace amounts of As, additionally in complex sample matrices, the direct determination of As species is difficult, and therefore, preliminary pre-treatment comprising the separation and/or pre-concentration of these species is highly desirable. Accordingly, in the sample preparation step, one or more As species (typically inorganic) can be separated through different extraction techniques. Early evolved arsines, both inorganic and methylated, can also be isolated according to their boiling points with the cryo-trapping (CT) technique 
or by the pervaporation-based membrane separation technique. Both approaches provide an excellent improvement in LODs of As species to $\leq \mathrm{ng} \mathrm{L}^{-1}$ levels for the same detector and improve the selectivity of measurements due to the alleviation of interference from sample matrix components.

\subsection{Pre-Concentration and Separation Methods before HG Process}

Selective complexation-extraction techniques, selective retention on solid adsorbents, i.e., solid phase extraction (SPE), and selective co-precipitations are the most common methods to separate/pre-concentrate As species, mainly inorganic forms, used in the sample preparation stage. Accordingly, As(III) or As(V) can be selectively extracted or co-precipitated with subsequent HG and detection. Total i-As is obtained after the initial conversion of As(III) $\leftrightarrow$ As(V), while the content of $\mathrm{As}(\mathrm{III})$ or $\mathrm{As}(\mathrm{V})$ can then be calculated by the difference. Non-chromatographic schemes for As speciation with the most popular pre-concentration/separation methods before HG, along with the achieved analytical performance, are summarized in Table S1.

\subsubsection{Selective Complexation-Extraction}

Among the various extraction techniques that could easily be adapted as the initial step in species-selective and -sensitive As determination combined with HG and spectrometric detection, cloud point extraction (CE), dispersive liquid-liquid microextraction (DLLME) and liquid-liquid extraction of aqueous two-phase systems (ATPS) are very popular.

Cloud point extraction, based on nonionic surfactants used as extracting solvents, offers many advantages like simplicity, safety, low cost and high pre-concentration factors. Different extraction procedures, making it possible to distinguish between i-As species, were proposed in methods developed to monitor As(III) and As(V) in natural water samples (drinking, tap, lake). For example, in one work [18], a selective complex of As(III) with Pyronine B in the presence of sodium dodecyl sulfate (SDS) at $\mathrm{pH} 10$ was extracted using a nonionic surfactant, Triton X-114. The surfactant-rich phase with As(III) was then separated and diluted with 1 mol L ${ }^{-1} \mathrm{HCl}$ prior to its determination by HG-AAS. Total i-As (As(III,V)) was extracted similarly after the pre-reduction of As(V) to As(III) with $\mathrm{Na}_{2} \mathrm{~S}_{2} \mathrm{O}_{3}$, and the $\mathrm{As}(\mathrm{V})$ content was calculated by the difference. In another work [23], As(III) and As(V) were separated by complexing with ammonium pyrrolidinedithiocarbamate (APDC) (at $\mathrm{pH} 4.6)$ and molybdate (at $\mathrm{pH} 2.4$ ), respectively, followed by quantitative extraction with Triton X-114. Afterwards, the As(III) content was determined by HG-AAS after diluting the surfactant-rich phase with $5 \% \mathrm{HCl}$. In the case of $\mathrm{As}(\mathrm{V})$, the resulting $\mathrm{As}(\mathrm{V})$ complex was first converted to free $\mathrm{As}(\mathrm{V})$ by ultrasonication, pre-reduced to As(III) with a thiourea-ascorbic acid mixture, and finally, determined by HG-AAS.

Dispersive liquid-liquid microextraction aims to extract the analytes from an aqueous phase into an organic phase, in which a third solvent is rapidly injected to accelerate efficient dispersion. Compared to traditional LE, it presents advantages in costs, labor, solvent consumption and enrichment factors. Chen et al. [30] applied the DLLME approach to speciate and quantify As(III) and As(V) in fruit juices in the presence of o-As (DMA and MMA) by HG-AFS using APDC (complexing agent), methanol (dispersant) and $\mathrm{CCl}_{4}$ (extractant). Samples were adjusted to $\mathrm{pH} 3$ and then mixed with APDC to form the As(III)-APDC complex, followed by injection of methanol and $\mathrm{CCl}_{4}$ to form a dispersion. After centrifugation, the organic phase with the As(III)-APDC complex was evaporated to dryness, and then the residue was dissolved in $1 \mathrm{~mol} \mathrm{~L}^{-1} \mathrm{HCl}$ and subjected to analysis by HG-AFS. Total i-As was determined after the pre-reduction of $\mathrm{As}(\mathrm{V})$ to $\mathrm{As}(\mathrm{III})$ using $\mathrm{Na}_{2} \mathrm{~S}_{2} \mathrm{O}_{3}$; next, the same protocol as for As(III) was used. Finally, the $\mathrm{As}(\mathrm{V})$ content was calculated from the difference. Under the selected pre-reduction and HG reaction conditions, limitations of the method for DMA were found to be advantageous to selectively measure i-As. In contrast, MMA contributed to the As response for $\mathrm{i}$-As. However, the degree of this interference was $\mathrm{pH}$-dependent; at $\mathrm{pH} 1.7-1.8$, the error was $<10 \%$.

A recent alternative to LE extraction is extraction by the aqueous two-phase system (ATPS). Its advantages include simplicity, low cost and high enrichment factors. These aqueous two-phase systems are primarily composed of water and other compounds of low toxicity. They can be formed by 
two heterogeneous phases composed of aqueous solutions of two incompatible polymers, i.e., a polymer and an electrolyte, or two types of physically incompatible electrolytes. Assis et al. [84] studied ATPS extraction comprising a polymer and an electrolyte prior to speciation of i-As forms in tap waters by HG-ICP-OES. The authors developed a procedure for the selective extraction of As(III) coexisting with $\mathrm{As}(\mathrm{V})$ using the ATPS composed of L64 (copolymers), water and $\mathrm{Na}_{2} \mathrm{SO}_{4}$ at $\mathrm{pH} 6.0$, while APDC was used to extract $\mathrm{As}(\mathrm{III})$; $\mathrm{As}(\mathrm{V})$ was poorly extracted in these conditions $(<18 \%)$.

\subsubsection{Selective Retention-Solid Phase Extraction}

Solid phase extraction is the most popular and commonly applied separation/pre-concentration technique. In SPE, analytes are extracted by sorption, eluted with a small amount of a solvent and then directly detected. Speciation analysis is realized by selective sorption or selective elution. SPE can fulfill the separation/pre-concentration requirements of As species before HG and spectrometric detection using a variety of sorbents. These could be conventional substances such as resins or gels (whose analytical capabilities could be further modified by surface modifications (functionalization) to improve the absorption performance), as well as alternative and novel ones like nanometer-sized materials or biosorbents with exceptional properties to effectively separate specific As species. Moreover, the method can easily be combined with different detection techniques in online $[9-11,25,26,37,55]$ or offline modes $[4,8,14,16,21,41,43,79]$.

The selection of appropriate sorbents is a key parameter in SPE. The development of alternative/novel sorbents is a general trend in pre-concentration and separation procedures for As speciation, whereas methodologies aimed at separating arsenate from arsenite are more widespread. Deng et al. [14] developed a simple and rapid method for the determination of trace amounts of total i-As in environmental water samples (ground, river, lake) by SPE on an aluminum hydroxide gel and HG-AFS detection. Trivalent arsenic was first oxidized to As(V) by $\mathrm{KMnO}_{4}$; then, the sample solution was adjusted to $\mathrm{pH}$ 6, followed by the addition of a freshly prepared gel for extraction of $\mathrm{As}(\mathrm{V})$. After centrifugation, the resultant precipitate with adsorbed i-As was dissolved in concentrated $\mathrm{HCl}$; then, As(V) was pre-reduced with a thiourea-ascorbic acid mixture, and finally, total i-As was determined as As(III) by HG-AFS. In two other works, SPE columns packed with cigarette filters [9] or PTFE particles [25] were used prior to HG and determination of the i-As content in various water samples (including tap, ground and seawater). The developed methods were based on selective online formation of the As(III)-APDC complex and its retention on SPE columns. As(V) did not form any complexes with APDC, and could not be retained in these conditions; hence, it passed through the columns. After reducing As(V) to As(III) with L-cysteine [9] or thiourea [25], the same system was applied to determine the total i-As, and $\mathrm{As}(\mathrm{V})$ was calculated by the difference. The adsorbed As(III)-APDC complex was online removed from SPE columns using $\mathrm{HCl}(1.7-2.0 \mathrm{~mol} \mathrm{~L}-1)$ and merged with $\mathrm{KBH}_{4}(2.1 \%)$ [9] or $\mathrm{NaBH}_{4}\left(4.1 \%\right.$ ) [25] solutions to generate $\mathrm{AsH}_{3}$ before entering AFS [9] or AAS [25] spectrometers for As(III) detection. Importantly, diluted $\mathrm{HCl}$ was used as the eluent because it also provided a favorable medium for the HG reaction.

In a few recent studies, graphene or carbon nanotubes have been used as sorbents for SPE. Considering As speciation, Khaligh et al. [4] presented an interesting approach to the speciation of i-As using nonporous graphene functionalized with carboxyl groups (G-COOH) for the ultrasound assisted, dispersive, micro-solid phase extraction (US-D- $\mu$-SPE) of As(V) from several natural water (tap, drinking, river, waste) and biological (human serum/urine) samples prior to its determination by HG-AAS. Briefly, As(V) was selectively retained on the G-COOH sorbent at $\mathrm{pH} 3.5$ by US-D- $\mu$-SPE with the next separation of the solid phase being achieved through centrifugation. Then, the As(V) retained on the sorbent was eluted with $\mathrm{NaOH}\left(0.3-0.5 \mathrm{~mol} \mathrm{~L}^{-1}\right)$, pre-reduced to As(III) with a KI-ascorbic acid mixture, and determined by HG-AAS. The previous oxidation of As(III) using $\mathrm{KMnO}_{4}$ made it possible to determine the total i-As. The difference between total i-As and As(V) yielded the As(III) content in the analyzed samples. The application of the carbon nanotube (CNT) sorbents for the determination of $\mathrm{As}(\mathrm{III})$ and $\mathrm{As}(\mathrm{V})$ at (ultra)trace levels in various environmental water samples (rain, snow, sea, 
river) is demonstrated in [10,26]. Wu et al. [10] packed a micropipette with single-walled (SW) CNTs to achieve the selective adsorption of the As(III)-APDC complex. The proposed speciation scheme involved the online formation and retention of the As(III)-APDC complex at $\mathrm{pH} 3$ on a SWCNTs-packed micro-column, followed by its online elution with $20 \% \mathrm{HNO}_{3}$ and determination of $\mathrm{As}$ (III) by HG-AFS using a sequential flow injection manifold. Total i-As was determined by the same protocol after pre-reduction of $\mathrm{As}(\mathrm{V})$ to $\mathrm{As}(\mathrm{III})$ with thiourea; $\mathrm{As}(\mathrm{V})$ was calculated by the difference. In contrast, to effectively improve the CNT material performance for a favorable selective adsorption of $\mathrm{As}(\mathrm{V})$ in the presence of As(III), Chen et al. [26] employed multi-walled (MW) CNTs functionalized with branched cationic polyethyleneimine (BPEI) that were packed into a mini-column for online SPE of $\mathrm{As}(\mathrm{V})$ in a sequential injection system following HG-AFS detection. Adsorption of $\mathrm{As}(\mathrm{V})$ was carried out at $\mathrm{pH} 5.8$, and the analyte was eluted with $0.6 \% \mathrm{NH}_{4} \mathrm{HCO}_{3}$. By following the same procedure, total i-As was determined after the oxidation of $\mathrm{As}(\mathrm{III})$ to $\mathrm{As}(\mathrm{V})$ with $\mathrm{H}_{2} \mathrm{O}_{2}$.

Other nanosized adsorbents for the separation and pre-concentration of As(III,V) species from natural water samples (tap, sea, ground, underground) were also found to be useful. For example, Erdogan et al. [16] synthesized nano-zirconium dioxide-boron oxide $\left(\mathrm{ZrO}_{2} / \mathrm{B}_{2} \mathrm{O}_{3}\right)$, called a "hybrid sorbent", and employed it for the selective sorption of $\mathrm{As}(\mathrm{V})$ by the SPE column technique prior to its determination by HG-AAS. In this SPE procedure, $\mathrm{As}(\mathrm{V})$ ions, retained at $\mathrm{pH}$ 3.0, were eluted with $3 \mathrm{~mol} \mathrm{~L}^{-1} \mathrm{HCl}$ and then pre-reduced to As(III) with a KI-ascorbic acid mixture and determined using HG-AAS. For total i-As, As(III) was oxidized firstly to As(V) by $\mathrm{KMnO}_{4}$ prior to SPE and then determined by HG-AAS; As(III) was calculated by the difference. To enhance the adsorption efficiency, Montoro Leal et al. [11] proposed magnetic nanoparticles (MNPs), i.e., ferrite $\left(\mathrm{Fe}_{3} \mathrm{O}_{4}\right)$, which were further functionalized with [1,5-bis (2-pyridyl) 3-sulfonophenylmethylene] thiocarbonohydrazine (PSTH-MNPs) and applied for the speciation of i-As by HG-ICP-MS. This procedure was based on the retention of $\mathrm{As}(\mathrm{III})$ and $\mathrm{As}(\mathrm{V})$ at $\mathrm{pH} 4$ in two knotted reactors filled with PSTH-MNPs, followed by the sequential elution of $\mathrm{As}(\mathrm{III})$ and total i-As in $7 \% \mathrm{HNO}_{3}-0.1 \%$ thiourea- $2.8 \%$ L-cysteine medium before HG using different $\mathrm{NaBH}_{4}$ concentration, i.e., 0.1 for $\mathrm{As}(\mathrm{III})$ and $0.5 \%$ for total i-As and measurement by ICP-MS. The concentration of $\mathrm{As}(\mathrm{V})$ was obtained by subtracting $\mathrm{As}(\mathrm{III})$ from total i-As.

Finally, strategies involving the use of biomaterials for SPE, including baker's yeasts (Saccharomyces cerevisiae) [8,37] or egg-shell membranes [21], were also proposed to speciate As species. Smichowski et al. [8] proposed a simple and sensitive method for the biosorption and pre-concentration of As(III) in the presence of $\mathrm{As}(\mathrm{V})$ in aqueous solutions using a batch system. A sample solution was combined with yeasts and $0.1 \mathrm{~mol} \mathrm{~L}^{-1}$ oxalic acid (acting as a reaction medium), adjusted to $\mathrm{pH} 7$ and then placed in a water bath $\left(60{ }^{\circ} \mathrm{C}, 30 \mathrm{~min}\right)$ to extract the As(III) form. After centrifugation, the solid phase was re-suspended in $4.0 \mathrm{~mol} \mathrm{~L}^{-1} \mathrm{HCl}$ to form a slurry, the liquid phase (supernatant) was acidified to $3.5 \mathrm{~mol} \mathrm{~L}^{-1} \mathrm{HCl}$, and $\mathrm{As}(\mathrm{III})$ and $\mathrm{As}(\mathrm{V})$ were determined correspondingly in both phases by HG-ICP-AES. To overcome possible matrix effects, the method of the standard addition was used for the determination of As(III) in suspension. Under selected conditions, As(III) was almost completely $(\sim 97 \%)$ retained by the biomass, likely bounded through -SH groups of yeast proteins, while As(V) remained in the supernatant. This made it possible to determine both As species in separate phases. Several different ground water samples were analyzed following the proposed method. In contrast, Koh et al. [37] showed that $\mathrm{As}(\mathrm{V})$ was retained better than $\mathrm{As}(\mathrm{III})$ in a yeast-immobilized column. In the cited work [37], S. cerevisiae was covalently bound onto controlled pore glass (CPG), packed into the column and used to selectively pre-concentrate $\mathrm{As}(\mathrm{V})$ over As(III). As a result, a simple flow injection system using the yeast-immobilized column coupled online with HG and ICP-AES for sensitive determinations of $\mathrm{As}(\mathrm{III})$ and $\mathrm{As}(\mathrm{V})$ was proposed. The manifold consisted of the SPE column and a manual injector. While the CPG-yeast column ( $\mathrm{pH} 7$ ) was loaded with the sample solution, As(III) was passed through the column, and hence, could be determined by HG-ICP-OES. Moving the injector to an alternative position, elution with $3 \mathrm{~mol} \mathrm{~L}^{-1} \mathrm{HNO}_{3}$ solution took place, releasing the $\mathrm{As}(\mathrm{V})$ retained on the column. The proposed method was applied for the determination of As species in herbicide, pesticide and cigarette samples. More recently, Zhang et al. [21] used a natural 
egg-shell membrane (ESM) as a sorptive material for SPE combined with HG-AFS to separate and determine $\mathrm{As}(\mathrm{V})$ in environmental water samples. The retention of $\mathrm{As}(\mathrm{V})$ on the ESM surface was via anion-exchange due to the presence of positively charged functional groups such as $-\mathrm{NH}_{3}{ }^{+}$and $-\mathrm{CO}-\mathrm{NH}_{2}{ }^{+}$. The ESM was obtained from fresh eggs and packed into a cartridge $(1 \mathrm{~g}, 6 \mathrm{~mL})$ by replacing its original C18 packing material. Sample solutions were adjusted to $\mathrm{pH} 11$, loaded on the ESM column, and then the cartridge was washed out with water and dried. The retained $\mathrm{As}(\mathrm{V})$ was eluted with 2 mol L ${ }^{-1} \mathrm{HNO}_{3}$ and measured by HG-AFS.

Regarding i-As, the application of SPE not only results in enhanced sensitivity due to the pre-concentration of the analytes; the separation of $\mathrm{i}$-As also plays a crucial role in minimizing interference coming from sample matrix compounds, further improving the selectivity of i-As measurements by HG. In this way, negative effects from hydride-active methylated As species (DMA and MMA) on activity of As(III,V) under reducing conditions are overcome. Successful applications of SPE include the selective determination of total i-As using anionic exchange or nonpolar resins [41-43,55,79] as sorbents. In three cited works [41,42,79], silica-based strong anion exchange (SAX) cartridges were used for the determination of i-As in rice [42], various rice products [41] and seafood samples [79]. They were used for offline SPE separation of i-As from DMA and MMA, followed by HG and spectrometric detection of the sum of As(III) and As(V). Species such as As(III), As(V), DMA and MMA were extracted with dilute $\mathrm{HNO}_{3}-\mathrm{H}_{2} \mathrm{O}_{2}$ solutions $(0.06-0.1 \mathrm{~mol} \mathrm{~L}-1,1-3 \%)$ to solubilize them and oxidize all i-As to As(V). Next, sample extracts were buffered (pH 5-7.5) and loaded onto SPE cartridges. Organic As species were washed out using an acetic acid solution $(0.1-0.5 \mathrm{~mol} \mathrm{~L}-1)$, and the retained $\mathrm{As}(\mathrm{V})$ ions were back-extracted (eluted) with $\mathrm{HCl}(0.5 \mathrm{~mol} \mathrm{~L}-1)$ [42,79] or $\mathrm{HNO}_{3}\left(0.4 \mathrm{~mol} \mathrm{~L}^{-1}\right)$ solutions [41]. Finally, As(V) was pre-reduced with a KI-ascorbic acid mixture and total i-As was measured in SPE eluates as As(III) by HG-AFS [42] or HG-AAS [41,79]. It is worth mentioning that the successful separation of i-As (as As(V)), via this SAX sorbent, was achieved through pH adjustment based on dissociation constants. Unfortunately, these methods were useless when the target analyte and matrix species had similar dissociation constants. To solve these problems, a novel method for separating i-As from other species by SPE was developed, including the chemical conversion of polar i-As to nonpolar compounds, such as $\mathrm{AsCl}_{3}$, which can be retained by a nonpolar resin. Accordingly, Huang et al. [43] employed a polystyrene (PS) resin to retain i-As from the matrix of rice as $\mathrm{AsCl}_{3}$. Arsenic species were extracted with $\mathrm{HNO}_{3}\left(0.02 \mathrm{~mol} \mathrm{~L}{ }^{-1}\right)$, and then sample extracts were acidified with $\mathrm{HCl}$ to $10 \mathrm{~mol} \mathrm{~L}^{-1}$, treated with thiourea to reduce $\mathrm{As}(\mathrm{V})$ into to As(III) and loaded on SPE cartridges. Then, cartridges were rinsed with $\mathrm{HCl}\left(10 \mathrm{~mol} \mathrm{~L}{ }^{-1}\right)$, and the retained $\mathrm{AsCl}_{3}$ was eluted by hydrolysis with water. Finally, HG-AFS was applied to quantify the concentration of total i-As as As(III) in SPE eluates. Similarly, Zhang et al. [55] reported online SPE using PS resin cartridges coupled with HG-AFS for the determination of $\mathrm{i}$-As in a complicated and arsenosugar-rich algae matrix. However, to fully overcome any matrix interferences and improve the retention efficiency of $\mathrm{i}-\mathrm{As}, \mathrm{Br}^{-}$ions were found to be more advantageous than $\mathrm{Cl}^{-}$ones for As(III) halogenation. In the proposed procedure, As species were initially extracted with $\mathrm{HClO}_{4}(1 \%)$ by a heat-vortex technique $\left(80^{\circ} \mathrm{C}, 20 \mathrm{~min}\right)$. Then, thiourea, $\mathrm{KBr}$ and $\mathrm{HCl}$ were added. In the presence of these reagents, $\mathrm{As}(\mathrm{V})$ was first reduced to As(III), and then i-As (as $\mathrm{As}(\mathrm{III})$ ) was converted into $\mathrm{AsBr}_{3}$, while the role of $\mathrm{HCl}$ was to maintain an acidic environment for the production of $\mathrm{AsBr}_{3}$ retained on the cartridge. The retained $\mathrm{AsBr}_{3}$ was eluted from the sorbent with water and total i-As was measured as As(III) by HG-AFS.

Otherwise, the separation o-As from i-As by SPE can be also applied only as a sample pre-treatment before analysis [23]. In this scenario, the examined water samples were passed through a glass column filled with small, activated $\mathrm{Al}_{2} \mathrm{O}_{3}$ to remove o-As. Adsorbed i-As species were desorbed by $0.2 \mathrm{~mol} \mathrm{~L}^{-1}$ $\mathrm{HCl}$, made up with water to a proper volume before subsequent analysis.

\subsubsection{Selective Co-Precipitation}

Arsenic species can also be selectively co-precipitated for separation. Van Elteren et al. [5] proposed a method for the determination of $\mathrm{As}(\mathrm{III})$ and $\mathrm{As}(\mathrm{V})$ in bottled mineral waters at ultratrace 
levels by HG-AFS coupled with a flow-injection system based on selective co-precipitation of As(III) with dibenzyldithiocarbamate (DBDTC) at low $\mathrm{pH}(\mathrm{pH}$ 2). The As-DBDTC precipitate was dissolved in $0.01 \mathrm{~mol} \mathrm{~L}^{-1} \mathrm{NaOH}$ and $30 \% \mathrm{H}_{2} \mathrm{O}_{2}$, and then $\mathrm{As}(\mathrm{III})$ was determined. The pre-reduction of $\mathrm{As}(\mathrm{V})$ to As(III) with a $\mathrm{KI}-\mathrm{K}_{2} \mathrm{~S}_{2} \mathrm{O}_{7}$ mixture was carried out before co-precipitation; this allowed the authors to determine total i-As and $\mathrm{As}(\mathrm{V})$, calculated by the difference.

\subsection{Pre-Concentration and Separation Methods after HG Process}

Another category of pre-concentration/separation methods is the trapping of As species hydrides in a cryogenic trap (CT) before their detection. This system (HG-CT) is a convenient approach to speciation of all hydride-active As species, i.e., inorganic and methylsubstituted, due to its pre-concentration and separation ability. Generally, non-selectively formed hydrides for various As species are first cryofocussed/trapped at liquid- $\mathrm{N}_{2}$ temperature in a U-shaped tube (acting as a separator of different hydrides), then sequentially released from the trap by its heating according to given boiling points (BP), i.e., $\mathrm{AsH}_{3}\left(-55^{\circ} \mathrm{C}\right.$, derived from $\left.\mathrm{As}(\mathrm{III}, \mathrm{V})\right), \mathrm{CH}_{3} \mathrm{AsH}_{2}\left(2{ }^{\circ} \mathrm{C}\right.$, derived from MMA $)$ and $\left(\mathrm{CH}_{3}\right)_{2} \mathrm{AsH}$ $\left(35.6{ }^{\circ} \mathrm{C}\right.$, derived from DMA), and finally transported to atomic spectrometers. To separate arsines in the trap, various types of packings were used; however, chromatographic materials are typically applied [24,61].

The main advantage of the HG-CT system is that analyses can be performed directly or with only a minimum sample pre-treatment, minimizing the risk of species inter-conversion. In view of this, an interesting approach to the determination of $\mathrm{i}-\mathrm{As}$, DMA and MMA in baby food (porridge powders and baby meals) by HG-CT-AAS, based on a slurry sampling using $3 \mathrm{~mol} \mathrm{~L}^{-1} \mathrm{HCl}$, was proposed [48]. Moreover, complex biological matrices such as human urine, cells, tissue or blood were simply lysed and/or diluted with water before analysis [31,60-63]. These minimally pretreated samples were then directly introduced into the HG device in the form of suspensions.

A remarkable quality of the HG-CT technique for As speciation is its ability to determine methylated three- and penta- valent As species in addition to inorganic ones. Here, the differentiation between inorganic and methylated $\mathrm{As}(\mathrm{III})$ - and $\mathrm{As}(\mathrm{V})$-species in the reaction with $\mathrm{NaBH}_{4}$ is based on $\mathrm{pH}$-dependent selective $\mathrm{HG}$ of arsines of As species of respective valences. Alternatively, it can be HG in the presence or absence of pre-reduction agents (usually L-cysteine), making it possible to overcome the different sensitivities of individual As species, as observed with the $\mathrm{pH}$-specific HG approach [60]. To overcome the problems associated with the low and narrow range of $\mathrm{HCl}$ concentrations required for HG reactions in the presence of L-cysteine, the use of buffered media, i.e., Tris- $\mathrm{HCl}$, was proposed. Furthermore, this approach also ensured a selective HG for the trivalent As species without its pre-reduction. This selective HG-CT approach was successfully combined with AAS and applied for analyses of different biological materials. Matousek et al. [60] and Hernandez-Zavala et al. [61] proposed oxidation state-specific speciation of inorganic and methylated arsenicals in complex biological matrices (e.g., cell cultures or tissue homogenates) by an automated HG-CT-AAS system equipped with a multiatomizer. Arsines for As(III,V), DMA(III,V) and MMA(III,V) were pre-concentrated and separated in a cryogenic chromatographic trap. To differentiate between As(III)and $\mathrm{As}(\mathrm{V})$-containing arsenicals, arsines for $\mathrm{As}(\mathrm{III})$-species were generated in the presence of a Tris- $\mathrm{HCl}$ buffer ( $\mathrm{pH}$ 6). Under the same conditions, a sample pre-treatment with L-cysteine allowed the authors to generate arsines for both $\mathrm{As}(\mathrm{III})-$ and $\mathrm{As}(\mathrm{V})$-species. Finally, the content of $\mathrm{As}(\mathrm{V})$-species was calculated as the difference. LODs of arsenicals ranged between $0.016-0.040 \mu \mathrm{g} \mathrm{L}^{-1}$ [61]. The proposed method was further improved by the online pre-reduction step integrated with the HG-CT system using TGA for pre-reduction [62]. The applicability of this method was also demonstrated in the case of As speciation in human urine samples. Replacing AAS with more sensitive AFS or ICP-MS detectors, and employing the identical HG-CT system, LODs of As species were significantly lowered to low $\mathrm{ng} \mathrm{L}^{-1}$ and sub ng L ${ }^{-1}$ levels. This permitted analyses to be performed of limited-size samples (e.g., tissue) or samples with an extremely low As content. Accordingly, Musil et al. [63] carried out speciation of inorganic and methyl-substituted arsenicals. i.e., As(III), i-As, DMA and MMA, 
in exfoliated bladder epithelial cells isolated from human urine by selective HG-CT-AFS with extremely low LODs of these As species, i.e., 0.00044, 0.00074, 0.00015 and $0.00017 \mu \mathrm{g} \mathrm{L}^{-1}$, respectively. In another work [31], the HG-CT-ICP-MS-based method was shown to be suitable for direct As speciation in whole blood and blood plasma at low exposure levels with LODs of $0 \mu \mathrm{g} \mathrm{L}^{-1}$ (i-As), $0.002 \mu \mathrm{g} \mathrm{L}-1$ (MMA) and $0.001 \mu \mathrm{L}^{-1}$ (DMA). For comparison, these LODs were about an order of magnitude lower than those achievable with the HG-CT-AAS system that was used for the analysis of the same samples, i.e., $0.15 \mu \mathrm{g} \mathrm{L}^{-1}$ (i-As), $0.09 \mu \mathrm{g} \mathrm{L}^{-1}$ (MMA) and $0.07 \mu \mathrm{g} \mathrm{L}{ }^{-1}$ (DMA).

Although a typical HG-CT system is equipped with a U-shaped tube, improvements to this trap have also been reported. For example, Hsiung and Wang [24] proposed a novel packed cold finger trap (PCFT) packed with a chromatographic material for the determination of As(III,V), DMA and MMA in fresh water and seawater by HG-AAS with a flame-heated, quartz-tube atomizer (QTA) under species-selective HG conditions. The advantage of this PCFT module over a typical U-shaped cryogenic trap lay in its better separation of collected arsines prior to their detection. In the proposed speciation scheme, As(III) was determined using a citrate buffer ( $\mathrm{pH}$ 6.4), and As(V) was assessed by subtracting the As(III) content from the total i-As content determined after pre-reduction of As(V) to As(III) with KI, while for DMA and MMA determination, compromised levels of chemical and instrumental parameters were selected. These parameters refer to the $\mathrm{HCl}-\mathrm{NaBH}_{4}$ concentrations for effective HG reactions, the flow rate of the carrier gas $(\mathrm{He})$ and the heating voltage of the PCFT device for reasonable separation between both methylated species. The achieved LODs were 0.047, 0.042, 0.0045 and $0.0063 \mu \mathrm{g} \mathrm{L}{ }^{-1}$ for As(III), As(V), MMA and DMA, respectively. More recently, Maratta et al. [19] developed a novel methodology for As speciation in ground and cistern water samples based on selective HG and CT on a CNTs-packed column, followed by elution of adsorbed arsines with 5\% $\mathrm{HNO}_{3}$ and their quantification by ETAAS. This speciation strategy involved the selective determination of $\mathrm{As}(\mathrm{III})$ and $\mathrm{As}(\mathrm{V})$, in addition to either inorganic (hydride-active) and organic (non-hydride-active) As fractions. As(III) was determined selectively using a citric buffer ( $\mathrm{pH} 4$ ), and As(III)+As(V) was determined after the pre-reduction of $\mathrm{As}(\mathrm{V})$ with L-cysteine and thiourea. In the case of the As organic fraction, it was decomposed into hydride-active species by UV photo-oxidation catalyzed by $\mathrm{TiO}_{2}$. This method yielded an enrichment factor of 60 and the LOD of As of $0.00078 \mu \mathrm{g} \mathrm{L}^{-1}$.

Interestingly, by combining two complementary techniques, i.e., HG-AAS and HG-CT-AAS, a complete experimental speciation protocol for the determination of As(III), As(V), MMA, DMA and non-hydride reactive As species at $\mathrm{ng} \mathrm{L}^{-1}$ levels in seawater was proposed [68]. In the first step, As was pre-concentrated by collecting the hydrides of As species into a graphite furnace before AAS detection. By selecting different $\mathrm{HG}$ reaction conditions, it was possible to determine As(III) alone (Tris- $\mathrm{HCl}, \mathrm{pH}$ ), total hydride reactive As species (after alkaline persulfate digestion), total As (after alkaline persulfate digestion submitted to an oxidative UV irradiation treatment of non-hydride reactive As species), and by the difference, non-hydride reactive As species. In the second step, hydrides of reactive As species were cryogenically trapped on a chromatographic column, followed by their sequential release and AAS determination in a heated quartz furnace. As a consequence, this system made it possible to separate and determine i-As, DMA and MMA.

Finally, hydrides of As species can be per-evaporated (P), i.e., separated from a sample matrix by simultaneous evaporation and gas diffusion through a membrane in a single step before performing measurements. In this manifold, the reactant mixture is transported to the lower chamber of a pervaporation unit from which the generated arsines evaporate first to the air gap between the liquid and the membrane. Then, they diffuse through the membrane into an acceptor stream (located in the acceptor chamber of this pervaporation module) for subsequent detection. Pervaporation appeared to be an excellent technique for the determination of inorganic As species in complex aqueous samples, known as "dirty", i.e., matrices containing an elevated content of organic matter or solid particles in suspension. Moreover, it allows direct analyses to be made of this kind of sample, as no prior pre-treatment such as filtration is required. Caballo-Lopez and de Castro [82] developed the HG-P-AFS method with the LOD of As of $0.42 \mu \mathrm{g} \mathrm{L}^{-1}$ to determine As(III) and As(V) in various "dirty" samples 
with suspended particulate matter. Selective HG was carried out by varying the $\mathrm{pH}$ at which arsines were generated, i.e., $\mathrm{pH} 1.3$ for $\mathrm{As}$ (III) and 0.2 for total i-As. Arsenic hydrides were generated in the reaction with $0.5 \% \mathrm{NaBH}_{4}$ and $6 \mathrm{~mol} \mathrm{~L}^{-1} \mathrm{HCl}$ in the absence of the pre-reduction step for total i-As. The concentration of $\mathrm{As}(\mathrm{V})$ was calculated as the difference. Importantly, under optimal conditions, the interference caused by DMA and MMA was negligible. In another work [86], the HG-P method coupled with CCD spectrophotometric detection was proposed for the selective determination of As(III) and $\mathrm{As}(\mathrm{V})$ in turbid river water samples with a high content of organic carbon. As(III) was selectively determined, generating $\mathrm{AsH}_{3}$ in the presence of a citrate buffer ( $\left.\mathrm{pH} 4.5\right)$. Both As(III) and As(V) were determined when HG was performed under highly acidic conditions $(\mathrm{pH}<1)$. The concentrations of the As species were calculated by simultaneously solving two proportional equations.

\section{Alternative HG Technique-Electrolytic Hydride Generation}

Undoubtedly, traditional $\mathrm{HG}$ using acid- $\mathrm{NaBH}_{4}$ for the species selective generation of As hydrides is the predominant method. Nevertheless, in recent years, interest in alternative techniques like electrolytic hydride generation (EcHG) has grown. The great advantage of the EcHG technique is that formation of hydride eliminates the use of the reducing $\mathrm{NaBH}_{4}$ reagent. In this technique, the reduction of the analyte to its hydride takes place on the cathode surface, and only acidic electrolyte solutions are required to ensure the electric current for the reduction process. Therefore, the efficiency of EcHG depends mainly on the cathode material and electrolytic current. Accordingly, Arbab-Zavar et al. [85] proposed a EcHG spectrophotometric method for the determination of As(III) and As(V) in tap water without pre-reduction of $\mathrm{As}(\mathrm{V})$. In the method, a graphite cathode was used to perform the reduction of $\mathrm{As}$ (III) to $\mathrm{AsH}_{3}$, while a $\mathrm{Sn} / \mathrm{Pb}$ alloy wire cathode was used to reduce $\mathrm{As}(\mathrm{V})$ to $\mathrm{AsH}_{3}$. Next, species were determined at $510 \mathrm{~nm}$ as the As(III)-SDDC complex obtained from the reaction between $\mathrm{AsH}_{3}$ and silver diethyldithiocarbamate (SDDC). The proposed method resulted in LODs of 20 (As(III)) and $60 \mu \mathrm{g} \mathrm{L}{ }^{-1}(\mathrm{As}(\mathrm{V}))$. Similarly, Li et al. [78] selectively determined i-As species by EcHG-AAS using a glassy carbon cathode in a $0.06 \mathrm{~mol} \mathrm{~L}^{-1} \mathrm{H}_{2} \mathrm{SO}_{4}$ catholyte medium. Differentiation between As(III) and $\mathrm{As}(\mathrm{V})$ was based on the different $\mathrm{HG}$ efficiencies attained by controlling electrolytic currents at 0.6 and 1.0 A, respectively. Concentrations of both species were calculated from slopes of calibration curves with LODs of $0.2 \mu \mathrm{g} \mathrm{L}^{-1}$ for As(III) and $0.5 \mu \mathrm{g} \mathrm{L}^{-1}$ for As(V). The method was successfully applied to speciate soluble i-As in Chinese medicines. Yang et al. [22] reported that the EcHG behavior of As(III), As(V), DMA and MMA could be changed at the modified graphite electrode (GE) by -SH modifiers including L-cysteine (Cys) and glutathione (GSH). The authors proposed a four-step analysis approach for the determination of all four As species in rice and natural water (river, lake, rain) samples by EcHG-AFS. Accordingly, As(III) was reduced on the GSH/GE at an applied current of 0.4 A. As(III), and $\mathrm{As}(\mathrm{V})$ generated $\mathrm{AsH}_{3}$ on the $\mathrm{Cys} / \mathrm{GE}$ at an applied current of 0.6 A. Measurements of As(III) and DMA were done on Cys/GE at two applied currents. Finally, the total As was determined after the reduction on Cys/GE at an applied current of 2.0 A. Under optimal conditions, LODs ranged between $0.10-0.25 \mu \mathrm{g} \mathrm{L}{ }^{-1}$. Lu et al. [58] demonstrated the application of a chemically modified carbon paste electrode (CMCPE) in EcHG to detect (ultra)trace amounts of As species. Given this, the authors developed a procedure for the selective determination of As(III) and total As in Chinese herbal medicines by EcHG-AFS. In the procedure, As(III), As(V), MMA and DMA were first selectively reduced to $\mathrm{AsH}_{3}$ at an applied current of 1.0 A on Cys/CMCPE. Then, under the same conditions, total As was determined after the pre-reduction of As(V), DMA, and MMA to As(III) with L-cysteine. Compared to traditional GE, the CMCPE provided better sensitivity; with CMCPE-AFS, LODs were successfully reduced to 0.095 (As(III)) and 0.087 (total As) $\mu \mathrm{g} \mathrm{L}^{-1}$.

\section{Conclusions}

In terms of toxicological investigations, knowledge of As species is crucial to understanding the potentially harmful effects associated with exposure to this element. HPLC-ICP-MS is undoubtedly the most popular coupled method for speciation analyses of As, because it provides a complete and 
sophisticated picture of species eluted from one injection of a sample, quantified at a (ultra)trace level. Nevertheless, as shown in this review, current research' interest is more focused on developing robust and reliable alternative methods obviating chromatographic separation. Non-chromatographic approaches to As speciation, based on the use of simple instrumentation which is available in most of laboratories (like atomic absorption or fluorescence detectors), are simpler, faster and economically friendly. They additionally provide degrees of sensitivity which are of the same order of magnitude or even better (related to possible species separation improving their pre-concentration) than those with hyphenated traditional chromatographic techniques using ICP-MS detection. In this sense, speciation achieved by selective HG prior to spectrometric detection is one of the most effective tools for distinguishing among four major toxic As species, i.e., As(III), As(V), DMA and MMA, or differentiating these toxic (hydride-active) species from non-toxic (non-hydride-active) arsenicals. In this way, this non-chromatographic approach gives sufficient information about As speciation for appropriate risk evaluations. Different physical-chemical properties of hydride-active As species (e.g., their volatility, redox potential) and the possibility of controlling experimental parameters such as $\mathrm{pH}$, reaction medium, reactant concentration, the presence of additives, temperature, etc., make it possible to develop species-selective non-chromatographic strategies for the speciation of trace amounts of different As species in a wide range of environmental, food and biological matrices. Furthermore, HG combined with various pre-concentration/separation approaches to accomplish As speciation serves as an excellent tool for the determination of As species at ultratrace levels. There is a belief that accurate (precise and true) methods for As speciation, which could be used for routine analyses of different samples, both with simple and complex matrices, will continue to develop.

The possibility of effectively speciating As using HG and atomic spectrometry gives rise to a willingness to further improve this technique, focused on reagent consumption, reduction, miniaturization and automation (employing various online flow systems). Simultaneously, as alternatives to chemical HG techniques, electrochemical (Ec)HG, for example, will be extensively studied and developed in the future, in an attempt to augment conventional HG for the determination and speciation of hydride forming elements.

Supplementary Materials: The following are available online, Table S1: Pre-concentration/separation methods to accomplish the speciation of inorganic As species by HG-based non-chromatographic techniques.

Author Contributions: Paper concept, data analysis and interpretation, writing, M.W.; paper preparation and visualization, A.S.-M., M.W.; discussion, A.S.-M., M.W., P.P.; review and editing, A.S.-M., P.P.; supervision, P.P.; funding acquisition, P.P. All authors have read and agreed to the published version of the manuscript.

Funding: This research received no external funding.

Acknowledgments: This work was financed by a statutory activity subsidy obtained by the Faculty of Chemistry, Wroclaw University of Science and Technology from the Polish Ministry of Science and Higher Education.

Conflicts of Interest: The authors declare no conflict of interest.

\section{References}

1. Caroli, S.; Torre, F.L.; Petrucci, F.; Violante, N. Arsenic speciation and Health Aspects. In Element Speciation in Bioinorganic Chemistry; Caroli, S., Ed.; John Wiley and Sons: New York, NY, USA, 1996; pp. 445-463.

2. Alp, O.; Tosun, G. A rapid on-line non-chromatographic hydride generation atomic fluorescence spectrometry technique for speciation of inorganic arsenic in drinking water. Food Chem. 2019, 290, 10-15. [CrossRef] [PubMed]

3. Bundaleska, J.M.; Stafilov, T.; Arpadjan, S. Direct analysis of natural waters for arsenic species by hydride generation atomic absorption spectrometry. Int. J. Environ. Anal. Chem. 2005, 85, 199-207. [CrossRef]

4. Khaligh, A.; Mousavi, H.Z.; Shirkhanloo, H.; Rashidi, A. Speciation and determination of inorganic arsenic species in water and biological samples by ultrasound assisted-dispersive-micro-solid phase extraction on carboxylated nanoporous graphene coupled with flow injection-hydride generation atomic absorption spectrometry. RSC Adv. 2015, 5, 93347-93359. 
5. van Elteren, J.T.; Stibilj, V.; Slejkovec, Z. Speciation of inorganic arsenic in some bottled Slovene mineral waters using HPLC-HGAFS and selective coprecipitation combined with FI-HGAFS. Water Res. 2002, 36, 2967-2974. [CrossRef]

6. Maity, S.; Chakravarty, S.; Thakur, P.; Gupta, K.K.; Bhattacharjee, S.; Roy, B.C. Evaluation and standardisation of a simple HG-AAS method for rapid speciation of $\mathrm{As}(\mathrm{III})$ and $\mathrm{As}(\mathrm{V})$ in some contaminated groundwater samples of West Bengal, India. Chemosphere 2004, 54, 1199-1206. [CrossRef]

7. Akter, K.F.; Chen, Z.; Smith, L.; Davey, D.; Naidu, R. Speciation of arsenic in ground water samples: A comparative study of CE-UV, HG-AAS and LC-ICP-MS. Talanta 2005, 68, 406-415.

8. Smichowski, P.; Marrero, J.; Ledesma, A.; Polla, G.; Batistoni, D.A. Speciation of As(III) and As(V) in aqueous solutions using baker's yeast and hydride generation inductively coupled plasma atomic emission spectrometric determination. J. Anal. At. Spectrom. 2000, 15, 1493-1497. [CrossRef]

9. Li, N.; Fang, G.; Zhu, H.; Gao, Z.; Wang, S. Determination of As(III) and As(V) in water samples by flow injection online sorption preconcentration coupled to hydride generation atomic fluorescence spectrometry. Microchim. Acta 2009, 165, 135-141. [CrossRef]

10. Wu, H.; Wang, X.; Liu, B.; Liu, Y.; Li, S.; Lu, J.; Tian, J.; Zhao, W.; Yang, Z. Simultaneous speciation of inorganic arsenic and antimony in water samples by hydride generation-double channel atomic fluorescence spectrometry with on-line solid-phase extraction using single-walled carbon nanotubes micro-column. Spectrochim. Acta B 2011, 66, 74-80. [CrossRef]

11. Montoro Leal, P.; Vereda Alonso, E.; López Guerrero, M.M.; Siles Cordero, M.T.; Cano Pavón, J.N.; García de Torres, A. Speciation analysis of inorganic arsenic by magnetic solid phase extraction on-line with inductively coupled mass spectrometry determination. Talanta 2018, 184, 251-259. [CrossRef]

12. Sigrist, M.E.; Beldomencio, H.R. Determination of inorganic arsenic species by flow injection hydride generation atomic absorption spectrometry with variable sodium tetrahydroborate concentrations. Spectrochim. Acta B 2004, 59, 1041-4045. [CrossRef]

13. Chaparro, L.L.; Ferrer, L.; Cerda, V.; Leal, L.O. Automated system for on-line determination of dimethylarsinic and inorganic arsenic by hydride generation-atomic fluorescence spectrometry. Anal. Bioanal. Chem. 2012, 404, 1589-1595. [CrossRef] [PubMed]

14. Deng, F.; Dong, R.; Yu, K.; Luo, X.; Tu, X.; Luo, S.; Yang, L. Determination of trace total inorganic arsenic by hydride generation atomic fluorescence spectrometry after solid phase extraction-preconcentration on aluminium hydroxide gel. Microchim. Acta 2013, 180, 509-515. [CrossRef]

15. Ciftci, T.D.; Henden, E. Arsenic Speciation of Waters from the Aegean Region, Turkey by Hydride Generation: Atomic Absorption Spectrometry. Bull. Environ. Contam. Toxicol. 2016, 97, 272-278. [CrossRef] [PubMed]

16. Erdogan, H.; Yalcınkaya, O.; Turker, A.R. Determination of inorganic arsenic species by hydride generation atomic absorption spectrometry in water samples after preconcentration/separation on nano $\mathrm{ZrO}_{2} / \mathrm{B}_{2} \mathrm{O}_{3}$ by solid phase extraction. Desalination 2011, 280, 391-396. [CrossRef]

17. Wang, N.; Tyson, J. Non-chromatographic speciation of inorganic arsenic by atomic fluorescence spectrometry with flow injection hydride generation with a tetrahydroborate-form anion-exchanger. J. Anal. At. Spectrom. 2014, 29, 665-673. [CrossRef]

18. Ulusoy, H.I.; Akcay, M.; Ulusoy, S.; Gürkan, R. Determination of ultra trace arsenic species in water samples by hydride generation atomic absorption spectrometry after cloud point extraction. Anl. Chim. Acta 2011, 703, 137-144. [CrossRef]

19. Maratta, A.; Martinez, L.D.; Pacheco, P. Development of an on line miniaturized non-chromatographic arsenic speciation system. Microchem. J. 2016, 127, 199-205. [CrossRef]

20. Anthemidis, A.N.; Zachariadis, G.A.; Stratis, J.A. Determination of arsenic(III) and total inorganic arsenic in water samples using an on-line sequential insertion system and hydride generation atomic absorption spectrometry. Anal. Chim. Acta 2005, 547, 237-242. [CrossRef]

21. Zhang, Y.; Wang, W.; Li, L.; Huang, Y.; Cao, J. Eggshell membrane-based solid-phase extraction combined with hydride generation atomic fluorescence spectrometry for trace arsenic $(\mathrm{V})$ in environmental water samples. Talanta 2010, 80, 1907-1912. [CrossRef]

22. Yang, X.-A.; Lu, X.-P.; Liu, L.; Chi, M.-B.; Hu, H.-H.; Zhang, W.-B. Selective determination of four arsenic species in rice and water samples by modified graphite electrode-based electrolytic hydride generation coupled with atomic fluorescence spectrometry. Talanta 2016, 159, 127-136. [CrossRef] [PubMed] 
23. Li, S.; Wang, M.; Zhong, Y.; Zhang, Z.; Yang, B. Cloud point extraction for trace inorganic arsenic speciation analysis in water samples by hydride generation atomic fluorescence spectrometry. Spectrochim. Acta B 2015, 111, 74-79. [CrossRef]

24. Hsiung, T.-M.; Wang, J.-M. Cryogenic trapping with a packed cold finger trap for the determination and speciation of arsenic by flow injection/hydride generation/atomic absorption spectrometry. J. Anal. At. Spectrom. 2004, 19, 923-928. [CrossRef]

25. dos Santos, Q.O.; Silva Junior, M.M.; Lemos, V.A.; Ferreira, S.L.C.; de Andrade, J.B. An online preconcentration system for speciation analysis of arsenic in seawater by hydride generation flame atomic absorption spectrometry. Microchem. J. 2018, 143, 175-180. [CrossRef]

26. Chen, M.; Lin, Y.; Gu, C.; Wang, J. Arsenic sorption and speciation with branch-polyethyleneimine modified carbon nanotubes with detection by atomic fluorescence spectrometry. Talanta 2013, 104, 53-57. [CrossRef]

27. Tasev, K.; Karadjova, I.; Stafilov, T. Determination of Inorganic and Total Arsenic in Wines by Hydride Generation Atomic Absorption Spectrometry. Microchim. Acta 2005, 149, 55-60. [CrossRef]

28. Karadjova, I.B.; Lampugnani, L.; Onor, M.; D’Ulivo, A.; Tsalev, D.L. Continuous flow hydride generation-atomic fluorescence spectrometric determination and speciation of arsenic in wine. Spectrochim. Acta B 2005, 60, 816-823. [CrossRef]

29. Cai, L.; Xu, C.; Zhong, M.; Wu, Y.; Zheng, S. Arsenic Speciation in Drinking Tea Samples by Hydride Generation Atomic Fluorescence Spectrometry. Asian J. Chem. 2013, 14, 8169-8172. [CrossRef]

30. Lai, G.; Chen, G.; Chen, T. Speciation of $A s^{\mathrm{III}}$ and $\mathrm{As}^{\mathrm{V}}$ in fruit juices by dispersive liquid-liquid microextraction and hydride generation-atomic fluorescence spectrometry. Food Chem. 2016, 190, 158-163. [CrossRef]

31. Matousek, T.; Wang, Z.; Douillet, C.; Musil, S.; Styblo, M. Direct Speciation Analysis of Arsenic in Whole Blood and Blood Plasma at Low Exposure Levels by Hydride Generation-Cryotrapping-Inductively Coupled Plasma Mass Spectrometry. Anal. Chem. 2017, 89, 9633-9637. [CrossRef]

32. Shi, J.-B.; Tang, Z.-Y.; Jin, Z.-X.; Chi, Q.; He, B.; Jiang, G.-b. Determination of As(III) and As(V) in soils using sequential extraction combined with flow injection hydride generation atomic fluorescence detection. Anal Chim. Acta 2003, 477, 139-147. [CrossRef]

33. Macedo, S.M.; de Jesus, R.M.; Garcia, K.S.; Hatje, V.; de Queiroz, S.A.F.; Ferreira, S.L.C. Determination of total arsenic and arsenic (III) in phosphate fertilizers and phosphate rocks by HG-AAS after multivariate optimization based on Box-Behnken design. Talanta 2009, 80, 974-979. [CrossRef] [PubMed]

34. Macedo, S.M.; dos Santos, D.C.; de Jesus, R.M.; da Rocha, G.O.; Ferreira, S.L.C.; de Andrade, J.B. Development of an analytical approach for determination of total arsenic and arsenic(III) in airborne particulate matter by slurry sampling and HG-FAAS. Microchem. J. 2010, 96, 46-49. [CrossRef]

35. do Nascimento, P.C.; Bohrer, D.; Becker, E.; de Carvalho, L.M. Comparison of different sample treatments for arsenic speciation in glass samples. J. Noncryst. Solids 2005, 351, 1312-1316. [CrossRef]

36. Rezende, H.C.; Coelho, N.M.M. Determination of Total Arsenic and Arsenic(III) in Phosphate Fertilizers by Hydride Generation Atomic Absorption Spectrometry After Ultrasound-Assisted Extraction Based on a Control Acid Media. J. AOAC Int. 2014, 97, 736-741. [CrossRef]

37. Koh, J.; Kwon, Y.; Pak, Y.-N. Separation and sensitive determination of arsenic species $\left(\mathrm{As}^{3+} / \mathrm{As}^{5+}\right)$ using the yeast-immobilized column and hydride generation in ICP-AES. Microchem. J. 2005, 80, 195-199. [CrossRef]

38. Cerveira, C.; Pozebon, D.; de Moraes, D.P.; de Fraga, J.C.S. Speciation of inorganic arsenic in rice using hydride generation atomic absorption spectrometry (HGAAS). Anal. Methods 2015, 7, 4528-4534. [CrossRef]

39. Matos Reyes, M.N.; Cervera, M.L.; Campos, R.C.; de la Guardia, M. Determination of arsenite, arsenate, monomethylarsonic acid and dimethylarsinic acid in cereals by hydride generation atomic fluorescence spectrometry. Spectrochim. Acta B 2007, 62, 1078-1082. [CrossRef]

40. Wang, Y.; Li, Y.; Lv, K.; Chen, X.; Yu, X. A simple and sensitive non-chromatographic method for quantification of four arsenic species in rice by hydride generation-atomic fluorescence spectrometry. Spectrochim. Acta B 2019, 149, 197-202. [CrossRef]

41. Rasmussen, R.R.; Qian, Y.; Sloth, J.J. SPE HG-AAS method for the determination of inorganic arsenic in rice-Results from method validation studies and a survey on rice products. Anal. Bioanal. Chem. 2013, 405, 7851-7857. [CrossRef]

42. Chen, G.; Chen, T. SPE speciation of inorganic arsenic in rice followed by hydride-generation atomic fluorescence spectrometric quantification. Talanta 2014, 119, 202-206. [CrossRef] 
43. Huang, Y.; Shan, J.; Fan, B.; He, Y.; Hia, S.; Sun, Y.; Lu, J.; Wang, M.; Wang, F. Determination of inorganic arsenic in rice by solid phase extraction and hydride generation atomic fluorescence spectrometry. Anal. Methods 2015, 7, 8896-8900. [CrossRef]

44. Musil, S.; Petursdottir, A.H.; Raab, A.; Gunnlaugsdottir, H.; Krupp, E.; Feldmann, J. Speciation without Chromatography Using Selective Hydride Generation: Inorganic Arsenic in Rice and Samples of Marine Origin. Anal. Chem. 2014, 86, 993-999. [CrossRef] [PubMed]

45. Welna, M.; Pohl, P.; Szymczycha-Madeja, A. Non-chromatographic Speciation of Inorganic Arsenic in Rice by Hydride Generation Inductively Coupled Plasma Optical Emission Spectrometry. Food Anal. Methods 2019, 12, 581-594. [CrossRef]

46. Schlotthauer, J.; Brusa, L.; Liberman, C.; Durand, M.; Livore, A.; Sigrist, M. Determination of inorganic arsenic in Argentinean rice by selective HGAAS: Analytical performance for paddy, brown and polished rice. J. Food Compos. Anal. 2020, 91, 103506. [CrossRef]

47. dos Santos, G.M.; Pozebon, D.; Cerveira, C.; de Moraes, D.P. Inorganic arsenic speciation in rice products using selective hydride generation and atomic absorption spectrometry (AAS). Microchem. J. 2017, 133, 265-271. [CrossRef]

48. Huber, C.S.; Vale, M.G.R.; Dessuy, M.B.; Svoboda, M.; Musil, S.; Dedina, J. Sample preparation for arsenic speciation analysis in baby food by generation of substituted arsines with atomic absorption spectrometry detection. Talanta 2017, 175, 406-412. [CrossRef]

49. Cava-Montesions, P.; de la Guardia, A.; Teutsch, C.; Cervera, M.C.; de la Guardia, M. Non-chromatographic speciation analysis of arsenic and antimony in milk hydride generation atomic fluorescence spectrometry. Anal. Chim. Acta 2003, 493, 195-203. [CrossRef]

50. Gonzalvez, A.; Llorens, A.; Cervera, M.L.; Armenta, S.; de la Guardia, M. Non-chromatographic speciation of inorganic arsenic in mushrooms by hydride generation atomic fluorescence spectrometry. Food Chem. 2009, 115, 360-364. [CrossRef]

51. Matos Reyes, M.N.; Cervera, M.L.; Campos, R.C.; de la Guardia, M. Non-chromatographic speciation of toxic arsenic in vegetables by hydride generation-atomic fluorescence spectrometry after ultrasound-assisted extraction. Talanta 2008, 75, 811-816. [CrossRef]

52. Cava-Montesinos, P.; Nilles, K.; Cervera, M.L.; de la Guardia, M. Non-chromatographic speciation of toxic arsenic in fish. Talanta 2005, 66, 895-901. [CrossRef] [PubMed]

53. Marschner, K.; Petursdottir, A.H.; Bucker, P.; Raab, A.; Feldmann, J.; Mester, Z.; Matousek, T.; Musil, S. Validation and inter-laboratory study of selective hydride generation for fast screening of inorganic arsenic in seafood. Anal. Chim. Acta 2019, 1049, 20-28. [CrossRef] [PubMed]

54. Petursdottir, A.H.; Gunnlaugsdottir, H. Selective and fast screening method for inorganic arsenic in seaweed using hydride generation inductively coupled plasma mass spectrometry (HG-ICPMS). Microchem. J. 2019, 144, 45-50. [CrossRef]

55. Zhang, W.; Qi, Y.; Qin, D.; Liu, J.; Mao, X.; Chen, G.; Wei, C.; Qian, Y. Determination of inorganic arsenic in algae using bromine halogenation and on-line nonpolar solid phase extraction followed by hydride generation atomic fluorescence spectrometry. Talanta 2017, 170, 152-157. [CrossRef]

56. Yang, L.-L.; Gao, L.-R.; Zhang, D.-Q. Speciation Analysis of Arsenic in Traditional Chinese Medicines by Hydride Generation-Atomic Fluorescence Spectrometry. Anal. Sci. 2003, 9, 897-902. [CrossRef]

57. Deng, T.; Liao, M.; Jia, M. Arsenic Speciation in Chinese Herb Ligusticum chuanxiong Hort by Hydride Generation Atomic Fluorescence Spectrometry. Spectrosc. Lett. 2005, 38, 109-119. [CrossRef]

58. Lu, X.-P.; Yang, X.-A.; Liu, L.; Hu, H.-H.; Zhang, W.-B. Selective and sensitive determination of As(III) and tAs in Chinese herbal medicine samples using L-cysteine modified carbon paste electrode-based electrolytic hydride generation and AFS analysis. Talanta 2017, 165, 258-266. [CrossRef]

59. Sun, H.; Liu, X.; Miao, Y. Speciation Analysis of Trace Inorganic Arsenic in Dietary Supplements by Slurry Sampling Hydride Generation Atomic Absorption Spectrometry. Food Anal. Methods 2011, 4, 251-257. [CrossRef]

60. Matousek, T.; Hernandez-Zavala, A.; Svoboda, M.; Langrova, L.; Adair, B.M.; Drobna, Z.; Thomas, D.J.; Styblo, M.; Dedina, J. Oxidation state specific generation of arsines from methylated arsenicals based on L-cysteine treatment in buffered media for speciation analysis by hydride generation-automated cryotrapping-gas chromatography-atomic absorption spectrometry with the multiatomizer. Spectrochim. Acta B 2008, 63, 396-406. 
61. Hernandez-Zavala, A.; Matousek, T.; Drobna, Z.; Paul, D.S.; Walton, F.; Adair, B.M.; Dedina, J.; Thomas, D.J.; Styblo, M. Speciation analysis of arsenic in biological matrices by automated hydride generation cryotrapping-atomic absorption spectrometry with multiple microflame quartz tube atomizer (multiatomizer). J. Anal. At. Spectrom. 2008, 23, 342-351. [CrossRef]

62. Musil, S.; Matousek, T. On-line pre-reduction of pentavalent arsenicals by thioglycolic acid for speciation analysis by selective hydride generation-cryotrapping-atomic absorption spectrometry. Spectrochim. Acta $B$ 2008, 63, 685-691. [CrossRef] [PubMed]

63. Musil, S.; Matousek, T.; Currier, J.M.; Styblo, M.; Dedina, J. Speciation Analysis of Arsenic by Selective Hydride Generation-Cryotrapping-Atomic Fluorescence Spectrometry with Flame-in-Gas-Shield Atomizer: Achieving Extremely Low Detection Limits with Inexpensive Instrumentation. Anal. Chem. 2014, 86, 10422-10428. [CrossRef] [PubMed]

64. Yu, H.; Li, C.; Tian, Y. Recent developments in determination and speciation of arsenic in environmental and biological samples by atomic spectrometry. Microchem. J. 2020, 152, 104312. [CrossRef]

65. Pohl, P. Hydride Generation-Recent Advances In Atomic Emission Spectrometry. TrAC Trends Anal. Chem. 2004, 23, 87-101. [CrossRef]

66. Marschner, K.; Musil, S.; Dedina, J. Demethylation of Methylated Arsenic Species during Generation of Arsanes with Tetrahydridoborate(1-) in Acidic Media. Anal. Chem. 2016, 88, 6366-6373. [CrossRef]

67. Carrero, P.; Malave, A.; Burguera, J.L.; Burguera, M.; Rondon, C. Determination of various arsenic species by flow injection hydride generation atomic absorption spectrometry: Investigation of the effects of the acid concentration of different reaction media on the generation of arsines. Anal. Chim. Acta 2001, 438, 195-204. [CrossRef]

68. Cabon, J.Y.; Cabon, N. Speciation of major arsenic species in seawater by flow injection hydride generation atomic absorption spectrometry. Fresenius J. Anal. Chem. 2000, 368, 484-489. [CrossRef]

69. Marschner, K.; Musil, S.; Miksik, I.; Dedina, J. Investigation of hydride generation from arsenosugars-Is it feasible for speciation analysis? Anal. Chim. Acta 2018, 1008, 8-17. [CrossRef]

70. Lehmann, E.L.; Fostier, A.H.; Arruda, M.A.Z. Hydride generation using a metallic atomizer after microwave-assisted extraction for inorganic arsenic speciation in biological samples. Talanta 2013, 104, 187-192. [CrossRef]

71. Welna, M.; Pohl, P. Potential of the hydride generation technique coupled to inductively coupled plasma optical emission spectrometry for non-chromatographic As speciation. J. Anal. At. Spectrom. 2017, 32, 1766-1779. [CrossRef]

72. Gonzales, J.C.; Lavilla, I.; Bendicho, C. Evaluation of non-chromatographic approaches for speciation of extractable As(III) and As(V) in environmental solid samples by FI-HGAAS. Talanta 2003, 59, 525-534.

73. Anwar, H.M. Arsenic speciation in environmental samples by hydride generation and electrothermal atomic absorption spectrometry. Talanta 2012, 88, 30-42.

74. Ramesh Kumar, A.; Riyazuddin, P. Non-chromatographic hydride generation atomic spectrometric techniques for the speciation analysis of arsenic, antimony, selenium, and tellurium in water samples-A review. Intern. J. Environ. Anal. Chem. 2007, 7, 469-500.

75. Pinheiro, B.S.; Gimenes, L.L.S.; Moreira, A.J.; Freschi, C.D.; Freschi, G.P.G. Arsenic Speciation in Environmental Samples Using Different Acid Concentrations and Ultrasonic Extraction for the Determination by HG-FAAS. At. Spectrosc. 2016, 37, 83-89.

76. Pinheiro, B.S.; Moreira, A.J.; Gimenes, L.L.S.; Freschi, C.D.; Freschi, G.P.G. UV photochemical hydride generation using $\mathrm{ZnO}$ nanoparticles for arsenic speciation in waters, sediments, and soils samples. Environ. Monit. Assess. 2020, 192, 331-343. [PubMed]

77. Petrov, P.K.; Serafimovska, J.M.; Arpadjan, S.; Tsalev, D.L.; Stafilov, T. Influence of EDTA, carboxylic acids, amino- and hydroxocarboxylic acids and monosaccharides on the generation of arsines in hydride generation atomic absorption spectrometry. Cent. Eur. J. Chem. 2008, 6, 216-221.

78. Li, X.; Jia, J.; Wang, Z. Speciation of inorganic arsenic by electrochemical hydride generation atomic absorption spectrometry. Anal. Chim. Acta 2006, 560, 153-158.

79. Rasmussen, R.R.; Hedegaard, R.V.; Larsen, E.H.; Sloth, J.J. Development and validation of an SPE HG-AAS method for determination of inorganic arsenic in samples of marine origin. Anal. Bioanal. Chem. 2012, 403, 2825-2834. [PubMed] 
80. Leal, L.O.; Forteza, R.; Cerda, V. Speciation analysis of inorganic arsenic by a multisyringe flow injection system with hydride generation-atomic fluorescence spectrometric detection. Talanta 2006, 69, 500-508. [PubMed]

81. Serafimovski, I.; Karadjova, I.B.; Stafilov, T.; Tsalev, D.L. Determination of total arsenic and toxicologically relevant arsenic species in fish by using electrothermal and hydride generation atomic absorption spectrometry. Microchem. J. 2006, 83, 55-60.

82. Caballo-Lopez, A.; Luque de Castro, M.D. Hydride generation-pervaporation-atomic fluorescence detection prior to speciation analysis of arsenic in dirty samples. J. Anal. At. Spectrom. 2002, 17, 1363-1367. [CrossRef]

83. Müller, J. Determination of inorganic arsenic(III) in ground water using hydride generation coupled to ICP-AES (HG-ICP-AES) under variable sodium boron hydride (NaBH4) concentrations. Fresenius J. Anal. Chem. 1999, 363, 572-576. [CrossRef]

84. Assis, R.C.; de Araujo Faria, B.A.; Caldeira, C.L.; Mageste, A.B.; de Lemos, L.R.; Rodrigues, G.D. Extraction of arsenic(III) in aqueous two-phase systems: A new methodology for determination and speciation analysis of inorganic arsenic. Microchem. J. 2019, 147, 429-436. [CrossRef]

85. Arbab-Zavar, M.H.; Chamsaz, M.; Heidari, T. Speciation and Analysis of Arsenic(III) and Arsenic(V) by Electrochemical Hydride Generation Spectrophotometric Method. Anal. Sci. 2010, 26, 107-110. [CrossRef] [PubMed]

86. Boonjob, W.; Miro, M.; Kolev, S.D. On-line speciation analysis of inorganic arsenic in complex environmental aqueous samples by pervaporation sequential injection analysis. Talanta 2013, 117, 8-13. [CrossRef] [PubMed]

Publisher's Note: MDPI stays neutral with regard to jurisdictional claims in published maps and institutional affiliations.

(C) 2020 by the authors. Licensee MDPI, Basel, Switzerland. This article is an open access article distributed under the terms and conditions of the Creative Commons Attribution (CC BY) license (http://creativecommons.org/licenses/by/4.0/). 